\title{
MFG-E8, A Novel Target of Promoting Osteogenic Differentiation of Human Bone Marrow Mesenchymal Stem Cells
}

\section{Jinwu Bai}

Zhejiang University School of Medicine Second Affiliated Hospital

\section{Weijun Zhang}

Zhejiang University School of Medicine Second Affiliated Hospital

\section{Kai Hang}

Zhejiang University School of Medicine Second Affiliated Hospital

\section{Guangfeng Zhao}

Zhejiang University School of Medicine Second Affiliated Hospital

\section{Huiming Zhong}

Zhejiang University School of Medicine Second Affiliated Hospital

\section{Chenwei Zhou}

Zhejiang University School of Medicine Second Affiliated Hospital

Jianxiang $\mathrm{Xu}$

Zhejiang University School of Medicine Second Affiliated Hospital

Wei Zhang

Zhejiang University School of Medicine Second Affiliated Hospital

\section{Erman Chen}

Zhejiang University School of Medicine Second Affiliated Hospital Jiaqi Wu

Zhejiang University School of Medicine Second Affiliated Hospital

\section{Ling Liu}

Hangzhou Hospital of Traditional Chinese Medicine

Deting Xue ( $\square$ blueskine@zju.edu.cn )

Zhejiang University School of Medicine Second Affiliated Hospital

\section{Research}

Keywords: MFG-E8, Osteogenic differentiation, hBMSCs, GSK3 $\beta / \beta$-catenin

Posted Date: September 21st, 2021

DOl: https://doi.org/10.21203/rs.3.rs-892581/v1 
License: (c) (i) This work is licensed under a Creative Commons Attribution 4.0 International License. Read Full License 


\section{Abstract}

\section{Background}

Fracture nonunion and bone defects are challenging for orthopedic surgeons. Milk fat globule-epidermal growth factor 8 (MFG-E8), a glycoprotein possibly secreted by macrophages in a fracture hematoma, participates in bone development. However, the role of MFG-E8 in the osteogenic differentiation of bone marrow mesenchymal stem cells (BMSCs) is unclear.

\section{Methods}

We investigated the osteogenic effect of MFG-E8 in vitro and in vivo. The CCK-8 assay was used to assess the effect of recombinant human MFG-E8 (rhMFG-E8) on the viability of human BMSCs (hBMSCs). Osteogenesis was investigated using real-time quantitative PCR, Western blotting, and immunofluorescence. Alkaline phosphatase (ALP) and Alizarin red staining were used to evaluate ALP activity and mineralization, respectively. An enzyme-linked immunosorbent assay was conducted to evaluate the secretory MFG-E8 concentration. Knockdown and overexpression of MFG-E8 in hBMSCs were established via siRNA and lentivirus vector transfection, respectively. Exogenous rhMFG-E8 was used to verify the in vivo therapeutic effect in a tibia bone-defect model based on radiographic analysis and histological evaluation.

\section{Results}

Endogenous and secretory MFG-E8 levels increased significantly during the early osteogenic differentiation of hBMSCs. Knockdown of MFG-E8 inhibited the osteogenic differentiation of hBMSCs. Overexpression of MFG-E8 and recombinant MFG-E8 protein increased the expression of osteogenesisrelated genes and proteins and enhanced calcium deposition. The active $\beta$-catenin to total $\beta$-catenin ratio and the $\mathrm{p}-\mathrm{GSK} 3 \beta$ protein level were increased by MFG-E8. The MFG-E8-induced enhanced osteogenic differentiation of hBMSCs was partially attenuated by a GSK3 $\beta / \beta$-catenin signaling inhibitor. Recombinant MFG-E8 accelerated bone healing in a rat tibial-defect model.

\section{Conclusions}

In conclusion, MFG-E8 promotes the osteogenic differentiation of hBMSCs by regulating the GSK3 $\beta / \beta$ catenin signaling pathway and so, is a potential therapeutic target.

\section{Introduction}

The healing of bone defects or fracture nonunion caused by high-energy injury, infection, tumor resection, and fracture are challenging for orthopedic surgeons [1-3]. The nonunion rate of all fractures ranges from $1.9-10 \%[4,5]$. Fracture healing is a complex and continuous process that includes hematoma formation and inflammatory responses as well as involves intracellular and extracellular signaling pathways $[6,7]$. The local hematoma formed after a fracture contains a variety of cells and cytokines and 
constitutes the fracture healing microenvironment, in which stem cells with osteogenic differentiation potential and cytokines affect the prognosis of the fracture[8, 9]. The fracture hematoma plays an important role in fracture healing[10]. However, the mechanism by which the fracture hematoma affects the osteogenic differentiation of bone marrow mesenchymal stem cells (BMSCs) is unclear.

Milk fat globule-epidermal growth factor 8 (MFG-E8), also known as lactadherin, is a secreted glycoprotein with a molecular weight of $46 \mathrm{kDa}[11]$. MFG-E8 is expressed in epithelial cells, macrophages, dendritic cells, and fibroblasts[12], It mediates the clearance of apoptotic cells[13] and the maintenance and repair of intestinal epithelial function [14], exerts an anti-inflammatory effect[15, 16], and regulates angiogenesis[17, 18] In the musculoskeletal system, osteoblasts and monocyte-derived osteoclasts express MFG-E8[19, 20]. MFG-E8-deficient mice exhibited increased RANKL-induced osteoclastogenesis ex vivo[21, 22]. Sinningen et al. also reported that MFG-E8-deficient mice exhibited impaired bone formation and mineralization compared with wild-type animals [23]. However, the role of MFG-E8 in the osteogenic differentiation of BMSCs is unclear. We investigated the effect of MFG-E8 on the osteogenic differentiation of human BMSCs (hBMSCs) and found that MFG-E8 promoted their osteogenic differentiation by regulating the GSK3 $\beta / \beta$-catenin signaling pathway in vitro and in vivo.

\section{Materials And Methods}

\section{Cell Culture, Regents and Antibodies}

The hBMSCs provided by Cyagen Biosciences (HUXMA-01001; Guangzhou, China) had the potential to differentiate into osteoblasts, chondrocytes, and adipocytes under appropriate conditions. Adherent hBMSCs were cultured in flasks with hBMSC growth medium (Cyagen Biosciences, Guangzhou, China) in an incubator at $37^{\circ} \mathrm{C}$ under $5 \% \mathrm{CO} 2$ and were passaged after reaching $80 \%$ confluence. The medium was replaced every 3 days; cells from passages $3-5$ was used in subsequent experiments.

Recombinant human MFG-E8 (rhMFG-E8) protein was purchased from R\&D Systems (Minneapolis, MN). Primary antibodies against GAPDH (CST\#5174), COL1A1 (CST\#72026), GSK3 $\beta$ (CST\#12456), and phospho-GSK-3 $\beta$ (Ser9) (CST\#5558) were purchased from Cell Signaling Technology (Danvers, MA). Primary antibodies against RUNX2 (ab192256), active $\beta$-catenin (ab246504), and total $\beta$-catenin (ab223075) were obtained from Abcam (Cambridge, UK). AR-A014418, a competitive and selective ATP inhibitor of GSK3 3 , was purchased from Selleck Chemicals (Houston, TX).

\section{Cell proliferation assay}

To assess the effects of rhMFG-E8 on hBMSC proliferation and viability, the cells were seeded into 96-well plates (2000 cells/well) and allowed to adhere for $18 \mathrm{~h}$. The medium was exchanged for hBMSC growth medium with rhMFG-E8 $(0,0.1,1,10,100$, or $1000 \mathrm{ng} / \mathrm{mL})$ for 1,3 , or 5 days. The medium was next changed for $10 \%$ Cell Counting Kit-8 (CCK-8) solution (Dojindo, Kumamoto, Japan) in $100 \mu \mathrm{L}$ of low-sugar Dulbecco's modified Eagle's medium (L-DMEM) without fetal bovine serum (FBS) (Gibco, Waltham, MA) 
for $4 \mathrm{~h}$ at $37^{\circ} \mathrm{C}$. The absorbance at $450 \mathrm{~nm}$ (A450), which is proportional to the number of viable cells, was measured using a microplate reader (ELX808; BioTek, Winooski, VT).

\section{Osteogenic differentiation protocol}

For osteogenic differentiation, hBMSCs were cultured in osteogenic induction medium (OIM) consisting of L-DMEM, 10\% FBS, $100 \mathrm{lU} / \mathrm{mL}$ penicillin/streptomycin, $100 \mathrm{nM}$ dexamethasone, $0.2 \mathrm{mM}$ ascorbic acid, and $10 \mathrm{mM} \beta$-glycerophosphate. First, hBMSCs were cultured in hBMSC growth medium in 6- or 12-well cell-culture plates (Corning, Shanghai, China) at a density of $3 \times 104 / \mathrm{cm} 2$ and incubated at $37^{\circ} \mathrm{C}$ under $5 \%$ C02. After cells reached about $80-90 \%$ confluence, the culture medium was replaced with fresh OIM. Subsequently, the OIM was replaced every 2 days.

\section{Small Interfering RNA (siRNA) transfection targeting MFG- E8}

To knock down the expression of MFG-E8 in hBMSCs, small interfering RNA (siRNA) transfection was performed. siRNAs for the human MFG-E8 gene were purchased from GenePharma Inc. (Shanghai, China). The sequences were as follows: siRNA 1, GGUUUAUGCGAGGAGAUUUTT; siRNA 2, GCCUUAAUGGACACGAAUUTT; siRNA 3, CCCACAAGAAGAACUUGUUTT. hBMSCs were cultured in six-well plates for $18 \mathrm{~h}$ prior to siRNA transfection. The medium was replaced with Opti-MEMTM I Reduced Serum Medium (Thermo Fisher Technology Co., Ltd., China) with 20 nM targeting siRNA or negative control using Lipo6000 ${ }^{\mathrm{TM}}$ transfection reagent (Beyotime Biotechnology, Shanghai, China). After culturing for $6 \mathrm{~h}$ at $37^{\circ} \mathrm{C}$ under $5 \% \mathrm{CO} 2$, the medium was exchanged for fresh hBMSC growth medium. The MFG-E8 mRNA and protein levels were determined using real-time PCR (RT-PCR) and Western blotting.

\section{Lentiviral packaging and cell infection}

MFG-E8-overexpression lentiviral vector particles (MFG-E8-overexpression group, OE), and lentiviral GFP vector particles (MFG-E8-overexpression control group, OE-NC) were provided by Cyagen Biosciences. Approximately 50-60\% confluent hBMSCs were incubated with lentiviral particles and $5 \mu \mathrm{g} / \mathrm{mL}$ polybrene in hBMSC growth medium at a multiplicity of infection of 50 (the optimum according to GFP expression after lentiviral GFP particle infection). For infection, hBMSCs were incubated with lentiviral particles and polybrene $(5 \mu \mathrm{g} / \mathrm{mL})$ in hBMSC growth medium. After about $18 \mathrm{~h}$, the infection medium was exchanged for fresh growth medium. After 3 days, the cells were screened using puromycin $(4 \mu \mathrm{g} / \mathrm{mL})$ and passaged for use in subsequent experiments. The expression of MFG-E8 was detected using RT-PCR, Western blot, and immunofluorescence.

\section{ALP staining and ALP activity assay}

ALP staining was used to investigate early mineralization. For ALP staining, cells were fixed with $4 \%$ paraformaldehyde (Sangon Biotech, Shanghai, China) for $30 \mathrm{~min}$. The cells were then washed with double distilled water ( $\mathrm{ddH} 2 \mathrm{O})$ three times and stained using an Alkaline Phosphatase Color Development kit (Beyotime, Shanghai, China). ALP activity was determined using an ALP Activity Assay kit (Beyotime) according to the manufacturer's instructions. Briefly, cells were lysed for $1 \mathrm{~h}$ with 
radioimmunoprecipitation assay (RIPA) buffer. The appropriate amount of supernatant and $50 \mu \mathrm{L}$ of chromogenic substrate (para-nitrophenyl phosphate) were added to wells of a 96-well plate, to which testing buffer was also added to a volume of $100 \mu \mathrm{L}$. We prepared standard samples (para-nitrophenol $0.5 \mathrm{mM}$ ) to generate an ALP standard curve. Next, the 96 -well plate was incubated at $37^{\circ} \mathrm{C}$ for $5-10 \mathrm{~min}$. Finally, to each well was added $100 \mu \mathrm{L}$ of reaction termination solution to stop the reaction, and the A405 was measured using a microplate reader.

\section{Alizarin red staining and quantification assay}

Alizarin red staining (ARS; Cyagen Biosciences) was performed to assess late mineralization. For ARS, cells were fixed in $4 \%$ paraformaldehyde for $20 \mathrm{~min}$ at room temperature and subsequently washed three times with ddH2O. Finally, the cells were treated with Alizarin red stain $(0.5 \%, \mathrm{pH} 4.1-4.2)$ for $20 \mathrm{~min}$ and rinsed with distilled water. To quantify the staining intensity, stained mineralized nodules were incubated with $10 \%$ cetylpyridinium chloride (Sigma, Shanghai, China), the solution was collected, and the A570 was measured using a microplate reader.

\section{Western blot analysis}

Cells were lysed in RIPA buffer supplemented with proteasome and phosphatase inhibitors (Boster Biological Technology, Wuhan, China). Equal amounts of proteins were separated using $10 \%$ SDS-PAGE and transferred onto polyvinylidene fluoride membranes (Millipore, Shanghai, China). After blocking in $5 \%$ non-fat milk for $1 \mathrm{~h}$, the membranes were incubated overnight at $4^{\circ} \mathrm{C}$ with antibodies specific against GAPDH (1:10,000), COL1A1 (1:1,000), RUNX2 (1:1,000), active $\beta$-catenin $(1: 1,000 ;)$, total $\beta$-catenin $(1: 1,000)$, phospho-GSK-3 $\beta(1: 1,000)$, and total GSK3 $\beta(1: 1,000)$. A stripping method was used to measure two antibodies of identical molecular weight. After washing four times (5 min each) in Tris-buffered saline with $0.1 \%$ Tween 20 (TBST), the membranes were incubated with a horseradish peroxidaseconjugated secondary anti-mouse or anti-rabbit antibody (Boster Biological Technology) for $1 \mathrm{~h}$ at room temperature. After washing three times ( 5 min each) with TBST, proteins were detected using enhanced chemiluminescence blotting reagents (Millipore). Signal intensities were measured using a Bio-Rad XRS chemiluminescence detection system (Bio-Rad, Hercules, CA).

\section{Immunofluorescence assay}

Cells were cultured in 12-well plates with OIM. After the induction of osteogenesis, the cells were fixed in $4 \%$ paraformaldehyde for $15 \mathrm{~min}$ at room temperature, permeabilized in $0.1 \%$ Triton X-100 for $30 \mathrm{~min}$ and blocked in $5 \%$ bovine serum albumin for $60 \mathrm{~min}$. Fixed cells were washed and incubated overnight with anti-RUNX2 (1:500), anti-COL1A1 (1:500), or anti-active $\beta$-catenin (1:500) antibody. The cells were incubated with a fluorescence-conjugated secondary antibody (DyLight 647 Conjugate; Boster Biological Technology) for $2 \mathrm{~h}$, and nuclei were stained with 2-(4-amidinophenyl)-6-indolecarbamidine dihydrochloride (DAPI) (Beyotime) for $5 \mathrm{~min}$. Samples were observed under a fluorescence microscope (EU5888; Leica, Wetzlar, Germany).

\section{RNA isolation and real time quantitative PCR}


Total RNA was isolated from cells cultured with OIM using RNAiso reagent (TaKaRa Bio Inc., Dalian, China) and quantified by measuring the A260 (NanoDrop 2000; Thermo Fisher Scientific, Waltham, MA). First-strand cDNA was synthesized using PrimeScript RT Master Mix (TaKaRa Bio Inc.) according to the manufacturer's instructions. Total RNA $(\leq 1,000 \mathrm{ng})$ was reverse-transcribed into cDNA in a reaction volume of $20 \mu \mathrm{L}$ using a Double-Strand cDNA Synthesis kit (TaKaRa Bio Inc.). The levels of mRNAs encoding COL1A1, RUNX2, OCN, Osterix, OPN, ALP, and GAPDH were determined using the StepOnePlus Real-Time PCR System (Applied Biosystems Inc., Warrington, UK) and SYBR Premix Ex Taq (TaKaRa Bio Inc.) with the following program: $95^{\circ} \mathrm{C}$ for $30 \mathrm{~s}$ followed by 40 cycles of $95^{\circ} \mathrm{C}$ for $5 \mathrm{~s}$ and $60^{\circ} \mathrm{C}$ for $30 \mathrm{~s}$. GAPDH was used as an internal control and for normalization. DNA concentrations were calculated using the $2-\Delta \Delta C$ t method24.[24] The primers were synthesized by Sangon Biotech and are listed in Table 1.

\section{ELISA}

MFG-E8 levels in the hBMSC culture supernatants were assessed via enzyme-linked immunosorbent assay (ELISA) using the Human MFGE ELISA Kit (Boster Biological Technology) following the manufacturer's protocol.

\section{In vivo evaluation in animals}

All animal experiments and procedures were conducted in accordance with the principles of the Institutional Animal Care Use Committee of the Second Affiliated Hospital of Zhejiang University and approved by the same committee. A rat tibial-defect model was used to assess the bone-forming ability of MFG-E8.[25, 26]. Fifteen rats were divided randomly into three groups: the blank group, normal saline (NS) (negative control group treated with NS) group, and rhMFG-E8 group. First, rats were anesthetized via inhalation of $2-5 \%$ isoflurane, with the anesthesia maintained via $2 \%$ isoflurane inhalation during surgery. After anesthesia, an incision was made lateral to the tibia, away from the bone. An intramedullary fixation pin (1.2-mm-diameter stainless-steel syringe needle) was inserted inside the medullary canal of the tibia for fixation. Osteotomy to create a transverse 1.5-mm-wide defect approximately $7 \mathrm{~mm}$ from the proximal tibial growth plate was performed using an electronic saw. The same leg was used in each group. Next, rhMFG-E8 $(20 \mu \mathrm{g})$ was injected locally at the fracture site on days $0,3,5,8$, and 11 (i.e., immediately and at 72-h intervals thereafter); NS was used as a vehicle control. Rats were sacrificed at 1 month after surgery, and samples were collected and fixed in $4 \%$ paraformaldehyde for $72 \mathrm{~h}$ at room temperature.

\section{Radiographic analysis}

A range of $3 \mathrm{~mm}$ above and below the bone-defect area of tibia samples was scanned using the $\mu \mathrm{CT}-100$ Imaging System (Scanco Medical, Brüttisellen, Switzerland) with the following parameters: $70 \mathrm{kVp}$; reconstruction matrix, 1,024; slice thickness, $14.8 \mu \mathrm{m}$; and exposure time, $300 \mathrm{~ms}$. The trabecular bone volume fraction (BV/TV), mean trabecular thickness (Tb.Th), mean trabecular number (Tb.N), and mean trabecular separation (Tb.Sp) were evaluated via standard three-dimensional microstructural analysis[25, 27]. 


\section{Histological evaluation}

After micro-computed tomography (CT), samples were decalcified using 10\% ethylene diamine tetra acetic acid (Sigma) in $0.1 \mathrm{M}$ phosphate-buffered saline, with the solution changed once a week for 6 weeks, before embedding in paraffin. Serial sections of $3 \mu \mathrm{m}$ thickness were cut and mounted on polylysine-coated slides and deparaffinized. The consecutive tissue sections were stained with hematoxylin and eosin (HE) or Masson's trichrome stain. $[25,28]$ Images were obtained using a microscope (Leica DM4000B; Leica, Wetzlar, Germany).

\section{Data and statistical analysis}

Statistical analysis was performed using Prism (version 8.0; GraphPad Software, San Diego, CA). All experiments were conducted at least three times and the data are presented as the means \pm SDs.

Differences between two groups were analyzed using the two-tailed Student's t-test. For comparisons of more than two groups, one-way analysis of variance followed by Bonferroni post hoc tests was used. In all analyses, $\mathrm{P}<0.05$ was taken to indicate statistical significance.

\section{Role of the funding source}

The funding agencies had no further role in study design, in the collection, analysis and interpretation of data, in the writing of the report and in the decision to submit the paper for publication.

\section{Results}

\section{Endogenous MFG-E8 expression increased significantly during osteogenic differentiation of hBMSCs}

To investigate the association between endogenous expression of MFG-E8 and osteogenic differentiation, we assayed MFG-E8 expression in undifferentiated and differentiated hBMSCs using RTPCR, Western blotting, and ELISA. Compared to levels in undifferentiated hBMSCs, MFG-E8 mRNA and protein levels increased significantly after osteogenic differentiation on days 1, 2, and 3. (Fig. 1A\&B) The secretory MFG-E8 protein level also increased significantly after osteogenic differentiation on days 1, 2, and 3 compared to undifferentiated hBMSCs (Fig. 1C). Therefore, endogenous MFG-E8 expression increased significantly during osteogenic differentiation of hBMSCs.

\section{Knockdown of MFG-E8 inhibited the osteogenic differentiation of hBMSCs and decreased calcium deposition}

To investigate the role of MFG-E8 in osteogenesis, three siRNAs were used to knock down MFG-E8 in hBMSCs. The efficiency of MFG-E8 knockdown was measured using RT-PCR. siRNA 1 and siRNA 2 resulted in significant gene knockdown, particularly siRNA2 (Fig. 2A). siRNA 2 also significantly decreased the MFG-E8 protein level (Fig. 2B) and so was used in subsequent experiments. 
To explore the effect of MFG-E8 knockdown on the osteogenesis of hBMSCs, osteogenesis-related genes and proteins were investigated using RT-PCR and Western blotting. The expression of osteogenesisrelated genes (RUNX2, Osterix, OCN, and ALP) was significantly decreased compared with the NC group after 1 and 3 days of osteogenesis (Fig. 2C\&D). The protein levels of RUNX2 and COL1A1 were decreased in the MFG-E8 siRNA group on days 1 and 3 of osteogenesis (Fig. 2E\&F). Moreover, significantly decreased ALP activity and mineral deposition were detected in the MFG-E8 siRNA group based on ALP staining and ARS (Fig. 2G).

\section{MFG-E8 overexpression increased the levels of osteogenesis-related proteins and enhanced ALP activity and calcium deposition}

To explore the role of endogenous MFG-E8 in the osteogenic differentiation of hBMSCs, MFG-E8 was overexpressed using lentiviral vectors. The efficiency of MFG-E8 overexpression was measured using RTPCR, Western blotting, and immunofluorescence. The MFG-E8 mRNA and protein levels increased significantly (Fig. 3A\&B), indicating successful MFG-E8 overexpression in hBMSCs.

The effect of endogenous MFG-E8 overexpression on osteogenesis was investigated. Compared to the OE-NC group (MFG-E8 overexpression control), the expression of osteogenesis-related genes such as RUNX2, COL1A1, Osterix, OCN, and ALP was significantly elevated in the OE group (MFG-E8 overexpression) on day 1 of osteogenesis (Fig. 3C). Western blotting showed that the RUNX2 and COL1A1 protein levels were increased in the OE group compared with the OE-NC group (Fig. 3D). ALP staining and ARS showed significantly increased ALP activity and calcium deposition in the OE group (Fig. 3E).

\section{Exogenous MFG-E8 increased the expression levels of osteogenesis-related genes and proteins and enhanced calcium deposition}

To assess the effect of exogenous MFG-E8 on osteogenic differentiation, BMSCs were cultured with OIM with rhMFG-E8 protein $(0,10,100$, and $1000 \mathrm{ng} / \mathrm{mL})$. Cell viability was unaffected by rhMFG-E8 (Fig. S1). The expression of osteogenesis-related genes (RUNX2, COL1A1, Osterix, OPN, and ALP) increased significantly as the rhMFG-E8 concentration increased on days 1 and 3 of osteogenesis compared with the control group (Fig. 4A). Additionally, the expression of osteogenesis-related proteins (RUNX2 and COL1A1) was elevated relative to levels in the control group on days 1 and 3 of osteogenesis in a concentration-dependent manner (Fig. 4B). These results were confirmed in the immunofluorescence assays (Fig. 4C).

We investigated the influence of rhMFG-E8 on early-stage mineralization during osteogenic differentiation. MFG-E8 significantly enhanced ALP activity on day 2 in a dose-dependent manner 
(Fig. 4D\&F). Based on ARS, MFG-E8 significantly increased calcium deposition and mineralization on day 11 (Fig. 4E\&G).

\section{MFG-E8 promotes the osteogenic differentiation of hBMSCs via the GSK3 $\beta / \beta$-catenin signaling pathway}

We evaluated the canonical Wnt/ $\beta$-catenin signaling pathway, which is vital in the differentiation of BMSCs into osteoblasts. Related proteins, including GSK3 $\beta$ and $\beta$-catenin, were measured using Western blotting on day 1 of osteogenesis. The expression of p-GSK3 $\beta$ and active $\beta$-catenin (a- $\beta$-catenin) was increased by MFG-E8, whereas the expression of total GSK3 $\beta$ and total $\beta$-catenin (t- $\beta$-catenin) was unaffected (Fig. 5A\&B). Furthermore, the ratio of a- $\beta$-catenin to t- $\beta$-catenin was increased significantly by GA (Fig. 5B). Immunofluorescence showed that the expression of a- $\beta$-catenin in the nucleus and cytoplasm was significantly increased by MFG-E8 (Fig. 5 C). Therefore, MFG-E8 promotes the osteogenic differentiation of hBMSCs via the GSK3 $\beta / \beta$-catenin signaling pathway.

\section{Enhanced osteogenic differentiation of hBMSCs caused by MFG-E8 was partially attenuated by a GSK3 $\beta / \beta$-catenin signaling inhibitor}

AR-A014418 is a competitive and selective ATP inhibitor of GSK3 $\beta$. The effect of AR-A014418 on GSK3 $\beta$ was explored using Western blotting. AR-A014418 at 10 and $20 \mu \mathrm{M}$ significantly decreased p-GSK3 $\beta$ and a- $\beta$-catenin expression but did not alter that of total GSK3 $\beta$ and t- $\beta$-catenin (Fig. 6A). Therefore, $20 \mu \mathrm{M}$ ARA014418 was used in subsequent experiments.

AR-A014418 attenuated the MFG-E8-induced increases in RUNX2 and COL1A1 expression (1000 ng/mL) on day 1 of osteogenesis, as determined by Western blotting (Fig. 6B). Furthermore, the MFG-E8-induced increases in levels of Wnt signaling pathway-related proteins such as p-GSK3 $\beta$ and a- $\beta$-catenin were attenuated significantly (Fig. 6B). Immunofluorescence showed that the increased expression of RUNX2 and COL1A1 caused by MFG-E8 was decreased significantly by AR-A014418 (Fig. 6C). In addition, the enhanced mineralization and ALP activity induced by MFG-E8 were attenuated by AR-A014418, as determined by ARS and the ALP assay (Fig. 6D-G).

\section{MFG-E8 accelerated bone healing in a rat tibial-defect model}

To investigate the role of MFG-E8 in vivo, exogenous recombinant MFG-E8 protein was administered to rats with tibial defects. Micro-CT showed that MFG-E8 significantly accelerated bone fracture healing compared to the control and NS groups. MFG-E8 treatment significantly reduced the gap distance of cortical defects in comparison with the other two groups (Fig. 7A). Compared with the control and NS groups, the MFG-E8 group had significantly higher BV/TV, Tb.N, and Tb.Th values and a lower Tb.Sp 
value (Fig. 7B). Histological analysis using HE and Masson's trichrome staining demonstrated better cortical growth in the MFG-E8 group compared with the other two groups (Fig. 7C).

\section{Discussion}

To our knowledge, this is the first report demonstrating the promotion of osteogenic differentiation of hBMSCs by MFG-E8 through the GSK3 $\beta / \beta$-catenin signaling pathway. The levels of endogenous and secretory MFG-E8 increased significantly during osteogenic differentiation of hBMSCs. Therefore, MFGE8 may be crucial for osteogenesis. Knockdown of MFG-E8 in hBMSCs inhibited their osteogenic differentiation and calcium deposition. Also, the overexpression of MFG-E8 or addition of exogenous rhMFG-E8 protein significantly increased the levels of osteogenesis-related genes and proteins, such as RUNX2, COL1A1, Osterix, ALP, OCN, and OPN, in hBMSCs. Furthermore, MFG-E8 increased ALP activity and calcium deposition, implying the enhancement of early and late osteogenesis. MFG-E8 promoted the osteogenic differentiation of hBMSCs by activating the Wnt/ $\beta$-catenin pathway. MFG-E8 inhibited GSK3 $\beta$ via phosphorylation, thus increasing the a- $\beta$-catenin level and inducing its translocation into the nucleus to activate gene transcription. Therefore, MFG-E8 promotes the osteogenic differentiation of hBMSCs by regulating $G S K 3 \beta / \beta$-catenin signaling pathway.

Endogenous and secretory MFG-E8 protein levels increased significantly during the osteogenic differentiation of hBMSCs. This suggests that MFG-E8 plays an important role in the osteogenic differentiation of hBMSCs. MFG-E8 is expressed ubiquitously in various tissues and cells[12, 29], and is universally distributed in mammals [12]. Therefore, the secretion and expression of MFG-E8 likely have complex regulatory effects. In the musculoskeletal system, MFG-E8 is expressed by osteoblasts, monocytes, and stromal cells $[19,20]$. Abe et al.[22] showed that MFG-E8 is expressed by and regulates osteoclasts, giant multinucleated cells that resorb bone during bone remodeling. The maintenance of bone homeostasis requires tight collaboration between osteoclasts and osteoblasts[30]. We found that when MFG-E8 expression is increased, it regulates the osteogenic differentiation of hBMSCs. The increase in MFG-E8 may be due to a positive feedback regulatory mechanism and may play other important regulatory roles in the osteogenic differentiation of hBMSCs.

MFG-E8 promoted the osteogenic differentiation of hBMSCs by activating the Wnt/ $\beta$-catenin pathway. The canonical Wnt/ $\beta$-catenin signaling pathway plays a key role in healing and promotes osteoblast function[31]. Activation of the Wnt pathway leads to the recruitment, phosphorylation, and inactivation of GSK3 $\beta$, resulting in cytoplasmic $\beta$-catenin stabilization and its translocation into the nucleus. This results in the transcription of downstream osteogenesis-related genes and the promotion of osteogenic differentiation of BMSCs[32-34]. We found significantly increased expression of phosphorylated GSK3 $\beta$ and a- $\beta$-catenin. Immunofluorescence revealed a high level of $\beta$-catenin in the nucleus. Inactivation (phosphorylation) of GSK3 $\beta$ is an intermediate step after activation of the Wnt/ $\beta$-catenin pathway[35, 36]. GSK3 $\beta$ plays important roles in the growth factor, hedgehog, G-protein coupling ligand, cytokine, and Wnt pathways [37]. The activation or inactivation of GSK3 $\beta$ is involved in multiple signaling pathways, such as that centered on PI3K-AKT. The activation of PI3K/AKT signaling can inhibit GSK3 $\beta$ via its 
phosphorylation at Ser9. However, MFG-E8 did not significantly alter the expression of p-AKT during osteogenesis (Fig. S2.0). Therefore, other molecules or signaling pathways may be involved in the promotion of osteogenic differentiation by MFG-E8 via the GSK3 $\beta / \beta$-catenin pathway.

Fracture hematomas were first reported to play an important role in fracture healing in the first half of the 20th century. Removal of the hematoma at the fracture site may delay fracture healing, and subcutaneous implantation of the hematoma can lead to heterotopic ossification[38, 39]. However, their role in the initiation of regeneration is unclear. Little is known about the initial cellular and humoral composition of the fracture hematoma $[39,40]$. Wray et al.[41] found increased $\beta$ - and $\gamma$-globulin in fracture hematoma supernatant. The increase in $\beta$-globulin may be due to hemoglobin, whereas the elevated $y$-globulin fraction may indicate the involvement of immune cells. The fracture hematoma microenvironment contains various immune cells and cytokines[42]. The immune system influences fracture healing while fractures influence systemic immunity $[43,44]$. Macrophages are the most important immune cells in osteoimmunology [45]. Laplante et al.[46] found that MFG-E8 secreted by apoptotic endothelial and epithelial cells reprograms macrophages from an M1 (proinflammatory) to an M2 (anti-inflammatory) phenotype and promotes wound healing. Therefore, MFG-E8 may maintain bone homeostasis by regulating inflammation and immunity via various cell types, such as macrophages and lymphocytes.

This study has several limitations. First, we only explored the role of the canonical Wnt/ $\beta$-catenin signaling pathway in regulating osteogenic differentiation by MFG-E8. Therefore, exploration of the noncanonical Wnt/ $\beta$-catenin pathway and other potential links between Wnt/ $\beta$-catenin and other signaling pathways by which MFG-E8 promotes osteogenic differentiation and regulates bone homeostasis is needed. Second, MFG-E8 promoted the osteogenic differentiation of hBMSCs but inhibited RANKLmediated osteoclast differentiation. The relationship between osteoblasts and osteoclasts needs to be investigated further. Third, bone healing involves endochondral ossification and intramembranous ossification[47]. We investigated intramembranous ossification; however, the effect of rhMFG-E8 on endochondral ossification is unclear. Future studies using bone-defect models should involve larger bone defects.

\section{Conclusion}

MFG-E8 promotes the osteogenic differentiation of hBMSCs by regulating the GSK3 $\beta / \beta$-catenin signaling pathway and is thus a novel therapeutic target for bone homeostasis.

\section{Abbreviations}

hBMSCs, Human bone marrow-derived mesenchymal stem cells; MFG-E8, Milk fat globule-epidermal growth factor 8; ARS, Alizarin red staining; RANKL, receptor activator of nuclear factor KB ligand; ALP, Alkaline phosphatase; GAPDH, glyceraldehyde-3-phosphate dehydrogenase; COL1A1, collagen type I a 1chain; RUNX2, runt-related transcription factor 2; OCN, osteocalcin; (active $\beta$-catenin, non-phosphorylated 
(active) $\beta$-catenin; $t-\beta$-catenin, total $\beta$-catenin; $H \& E$, hematoxylin and eosin; BV/TV, trabecular bone volume per total volume; Tb.N, Trabecular Number ; Tb.Th, Trabecular thickness; Tb.Sp, Trabecular Separation;

\section{Declarations}

\section{Authors' contributions}

$\mathrm{JB}, \mathrm{WJZ}$ and $\mathrm{KH}$ designed research, performed research, analyzed data and wrote the paper. $\mathrm{GZ}, \mathrm{HZ}$ and JX performed research and wrote the paper. CZ, WZ, EC, JW and LL analyzed data and wrote the paper. DX: designed research, analyzed date wrote the paper and review \& editing, Supervision. And all authors listed have read and approved the manuscript that is enclosed.

\section{Declaration of competing interest}

The authors, their immediate families, and any research foundations with which they are affiliated have not received any financial payments or other benefits from any commercial entity related to the subject of this article. None of the authors have any conflicts of interest with regards to this research.

\section{Consent for publication}

Not applicable.

\section{Ethics approval and consent to participate}

All animal experiments were conducted in accordance with principles and procedures approved by the Institutional Animal Care Use Committee at the second affiliated hospital of Zhejiang University. All of the treatment procedures conformed to the ethical standards established in the 1964 Declaration of Helsinki.

This study was performed at the Second Affiliated Hospital of Zhejiang University School of Medicine, Hangzhou, Zhejiang, China.

\section{Funds}

This research was supported by the Joint Funds of the Zhejiang Provincial Natural Science Foundation of China (No. LBY21H060004), the Zhejiang Provincial Natural Science Foundation of China (No. LY18H060003), the National Natural Science Foundation of China (No. 81871759 and No. 82172189).

\section{Acknowledgements}

We thank all clients from the Clinical Research Center of the Second Affiliated Hospital, Zhejiang University, including Lingling Zhang, Jiayu Chen and Chongc Li et al.

\section{Availability of data and materials}


The datasets used and/or analyzed during the current study are available from the corresponding author on reasonable request.

\section{References}

1. Dimitriou R, Jones E, McGonagle D, Giannoudis PV. Bone regeneration: current concepts and future directions. BMC Med. 2011;9:66.

2. Verrier S, Alini M, Alsberg E, Buchman SR, Kelly D, Laschke MW, et al. Tissue engineering and regenerative approaches to improving the healing of large bone defects. Eur Cell Mater. 2016;32:87110.

3. El-Rashidy AA, Roether JA, Harhaus L, Kneser U, Boccaccini AR. Regenerating bone with bioactive glass scaffolds: A review of in vivo studies in bone defect models. Acta Biomater. 2017;62:1-28.

4. Zura R, Xiong Z, Einhorn T, Watson JT, Ostrum RF, Prayson MJ, et al. Epidemiology of Fracture Nonunion in 18 Human Bones. JAMA Surg. 2016;151:e162775.

5. Rupp M, Biehl C, Budak M, Thormann U, Heiss C, Alt V. Diaphyseal long bone nonunions - types, aetiology, economics, and treatment recommendations. Int Orthop. 2018;42:247-58.

6. Cho TJ, Gerstenfeld LC, Einhorn TA. Differential temporal expression of members of the transforming growth factor beta superfamily during murine fracture healing. J Bone Miner Res. 2002;17:513-20.

7. Einhorn TA. The cell and molecular biology of fracture healing. Clin Orthop Relat Res. 1998:S7-21.

8. Dong CH, Deng YS, Yang XJ, Liu J, Liu R, Hou FY, et al. The interplay of transcriptional and posttranscriptional regulation of migration of mesenchymal stem cells during early stages of bone fracture healing. Eur Rev Med Pharmacol Sci. 2017;21:5542-7.

9. Qiao Z, Greven J, Horst K, Pfeifer R, Kobbe P, Pape HC, et al. Fracture Healing and the Underexposed Role of Extracellular Vesicle-Based Cross Talk. Shock. 2018;49:486-96.

10. Ankrum JA, Ong JF, Karp JM. Mesenchymal stem cells: immune evasive, not immune privileged. Nat Biotechnol. 2014;32:252-60.

11. Yi YS. Functional Role of Milk Fat Globule-Epidermal Growth Factor VIII in Macrophage-Mediated Inflammatory Responses and Inflammatory/Autoimmune Diseases. Mediators Inflamm. 2016;2016:5628486.

12. Aziz M, Jacob A, Matsuda A, Wang P. Review: milk fat globule-EGF factor 8 expression, function and plausible signal transduction in resolving inflammation. Apoptosis. 2011;16:1077-86.

13. Hanayama R, Tanaka M, Miwa K, Shinohara A, Iwamatsu A, Nagata S. Identification of a factor that links apoptotic cells to phagocytes. Nature. 2002;417:182-7.

14. Bu HF, Zuo XL, Wang X, Ensslin MA, Koti V, Hsueh W, et al. Milk fat globule-EGF factor 8/lactadherin plays a crucial role in maintenance and repair of murine intestinal epithelium. $\mathrm{J}$ Clin Invest. 2007;117:3673-83.

15. Aziz MM, Ishihara S, Mishima Y, Oshima N, Moriyama I, Yuki T, et al. MFG-E8 attenuates intestinal inflammation in murine experimental colitis by modulating osteopontin-dependent alphavbeta3 
integrin signaling. J Immunol. 2009;182:7222-32.

16. Aziz M, Matsuda A, Yang WL, Jacob A, Wang P. Milk fat globule-epidermal growth factor-factor 8 attenuates neutrophil infiltration in acute lung injury via modulation of CXCR2. J Immunol. 2012;189:393-402.

17. Silvestre JS, Thery C, Hamard G, Boddaert J, Aguilar B, Delcayre A, et al. Lactadherin promotes VEGFdependent neovascularization. Nat Med. 2005;11:499-506.

18. Motegi S, Leitner WW, Lu M, Tada Y, Sardy M, Wu C, et al. Pericyte-derived MFG-E8 regulates pathologic angiogenesis. Arterioscler Thromb Vasc Biol. 2011;31:2024-34.

19. Han X, Bolcato AL, Amar S. Identification of genes differentially expressed in cultured human osteoblasts versus human fibroblasts by DNA microarray analysis. Connect Tissue Res. 2002;43:6375.

20. Harre U, Keppeler H, Ipseiz N, Derer A, Poller K, Aigner M, et al. Moonlighting osteoclasts as undertakers of apoptotic cells. Autoimmunity. 2012;45:612-9.

21. Sinningen K, Thiele S, Hofbauer LC, Rauner M. Role of milk fat globule-epidermal growth factor 8 in osteoimmunology. Bonekey Rep. 2016;5:820.

22. Abe T, Shin J, Hosur K, Udey MC, Chavakis T, Hajishengallis G. Regulation of osteoclast homeostasis and inflammatory bone loss by MFG-E8. J Immunol. 2014;193:1383-91.

23. Sinningen K, Albus E, Thiele S, Grossklaus S, Kurth T, Udey MC, et al. Loss of milk fat globuleepidermal growth factor 8 (MFG-E8) in mice leads to low bone mass and accelerates ovariectomyassociated bone loss by increasing osteoclastogenesis. Bone. 2015;76:107-14.

24. Livak KJ, Schmittgen TD. Analysis of relative gene expression data using real-time quantitative PCR and the 2(-Delta Delta C(T)) Method. Methods. 2001;25:402-8.

25. Zhang W, Chen E, Chen M, Ye C, Qi Y, Ding Q, et al. IGFBP7 regulates the osteogenic differentiation of bone marrow-derived mesenchymal stem cells via Wnt/beta-catenin signaling pathway. FASEB J. 2018;32:2280-91.

26. Hang K, Ye C, Xu J, Chen E, Wang C, Zhang W, et al. Apelin enhances the osteogenic differentiation of human bone marrow mesenchymal stem cells partly through Wnt/beta-catenin signaling pathway. Stem Cell Res Ther. 2019;10:189.

27. Chen EEM, Zhang W, Ye CCY, Gao X, Jiang LLJ, Zhao TTF, et al. Knockdown of SIRT7 enhances the osteogenic differentiation of human bone marrow mesenchymal stem cells partly via activation of the Wnt/beta-catenin signaling pathway. Cell Death Dis. 2017;8:e3042.

28. Bai J, Xu J, Hang K, Kuang Z, Ying L, Zhou C, et al. Glycyrrhizic Acid Promotes Osteogenic Differentiation of Human Bone Marrow Stromal Cells by Activating the Wnt/beta-Catenin Signaling Pathway. Front Pharmacol. 2021;12:607635.

29. Li BZ, Zhang HY, Pan HF, Ye DQ. Identification of MFG-E8 as a novel therapeutic target for diseases. Expert Opin Ther Targets. 2013;17:1275-85. 
30. Hang K, Ye C, Chen E, Zhang W, Xue D, Pan Z. Role of the heat shock protein family in bone metabolism. Cell Stress Chaperones. 2018;23:1153-64.

31. Krishnan V, Bryant HU, Macdougald OA. Regulation of bone mass by Wnt signaling. J Clin Invest. 2006;116:1202-9.

32. Glass DA, 2nd, Bialek P, Ahn JD, Starbuck M, Patel MS, Clevers $\mathrm{H}$, et al. Canonical Wnt signaling in differentiated osteoblasts controls osteoclast differentiation. Dev Cell. 2005;8:751-64.

33. Hill TP, Spater D, Taketo MM, Birchmeier W, Hartmann C. Canonical Wnt/beta-catenin signaling prevents osteoblasts from differentiating into chondrocytes. Dev Cell. 2005;8:727-38.

34. Mbalaviele G, Sheikh S, Stains JP, Salazar VS, Cheng SL, Chen D, et al. Beta-catenin and BMP-2 synergize to promote osteoblast differentiation and new bone formation. J Cell Biochem. 2005;94:403-18.

35. Lin FX, Zheng GZ, Chang B, Chen RC, Zhang QH, Xie P, et al. Connexin 43 Modulates Osteogenic Differentiation of Bone Marrow Stromal Cells Through GSK-3beta/Beta-Catenin Signaling Pathways. Cell Physiol Biochem. 2018;47:161-75.

36. Hur EM, Zhou FQ. GSK3 signalling in neural development. Nat Rev Neurosci. 2010;11:539-51.

37. Wu D, Pan W. GSK3: a multifaceted kinase in Wnt signaling. Trends Biochem Sci. 2010;35:161-8.

38. Mizuno K, Mineo K, Tachibana T, Sumi M, Matsubara T, Hirohata K. The osteogenetic potential of fracture haematoma. Subperiosteal and intramuscular transplantation of the haematoma. J Bone Joint Surg Br. 1990;72:822-9.

39. Park SH, Silva M, Bahk WJ, McKellop H, Lieberman JR. Effect of repeated irrigation and debridement on fracture healing in an animal model. J Orthop Res. 2002;20:1197-204.

40. Grundnes 0 , Reikeras 0 . The importance of the hematoma for fracture healing in rats. Acta Orthop Scand. 1993;64:340-2.

41. Wray JB. The biochemical characteristics of the fracture hematoma in man. Surg Gynecol Obstet. 1970;130:847-52.

42. Walters G, Pountos I, Giannoudis PV. The cytokines and micro-environment of fracture haematoma: Current evidence. J Tissue Eng Regen Med. 2018;12:e1662-e77.

43. Busti AJ, Hooper JS, Amaya CJ, Kazi S. Effects of perioperative antiinflammatory and immunomodulating therapy on surgical wound healing. Pharmacotherapy. 2005;25:1566-91.

44. Kolar P, Schmidt-Bleek K, Schell H, Gaber T, Toben D, Schmidmaier G, et al. The early fracture hematoma and its potential role in fracture healing. Tissue Eng Part B Rev. 2010;16:427-34.

45. Yang D, Wan Y. Molecular determinants for the polarization of macrophage and osteoclast. Semin Immunopathol. 2019;41:551-63.

46. Laplante P, Brillant-Marquis F, Brissette MJ, Joannette-Pilon B, Cayrol R, Kokta V, et al. MFG-E8 Reprogramming of Macrophages Promotes Wound Healing by Increased bFGF Production and Fibroblast Functions. J Invest Dermatol. 2017;137:2005-13. 
47. Frost HM. The biology of fracture healing. An overview for clinicians. Part I. Clin Orthop Relat Res. 1989:283-93.

\section{Table}

\section{TABLE I}

Sequences of primers for real-time quantitative PCR analysis

\begin{tabular}{|c|c|c|}
\hline Gene name & Forward Primer $\left(5^{\prime} \rightarrow 3^{\prime}\right)$ & Reverse Primer $\left(5^{\prime} \rightarrow 3^{\prime}\right)$ \\
\hline COL1A1 & CAGATCACGTCATCGCACAAC & GAGGGCCAAGACGAAGACATC \\
\hline RUNX2 & TGGTTACTGTCATGGCGGGTA & TCTCAGATCGTTGAACCTTGCTA \\
\hline Osterix & AGCCCATTAGTGCTTGTAAAGG & СCTCTGCGGGACTCAACAAC \\
\hline OCN & САСТССТCGСССTATTGGC & СССTCCTGCTTGGACACAAAG \\
\hline OPN & СTCCATTGACTCGAACGACTC & CAGGTCTGCGAAACTTCTTAGAT \\
\hline ALP & ACCACCACGAGAGTGAACCA & CGTTGTCTGAGTACCAGTCCC \\
\hline GAPDH & GGAGCGAGATCCCTCCAAAAT & GGCTGTTGTCATACTTCTCATGG \\
\hline
\end{tabular}

\section{Figures}

A

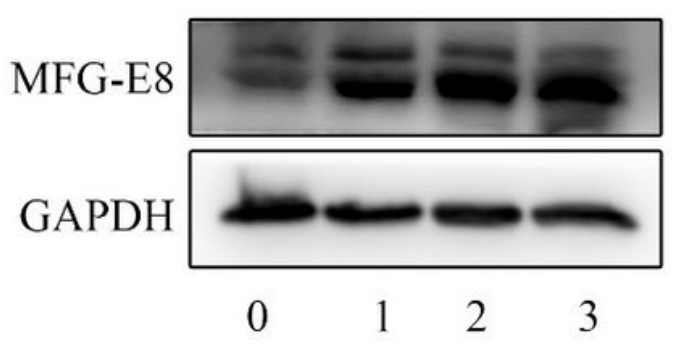

$\mathrm{B}$

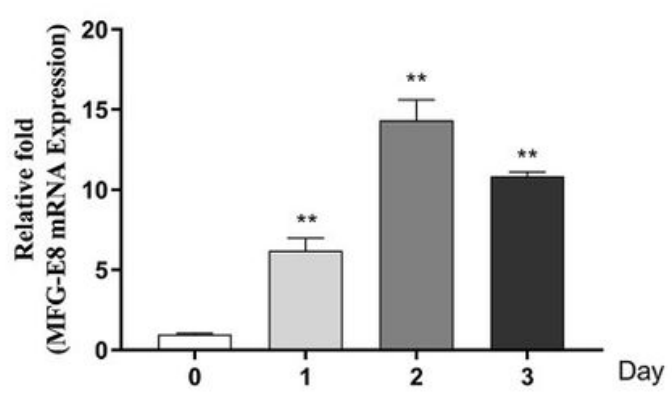

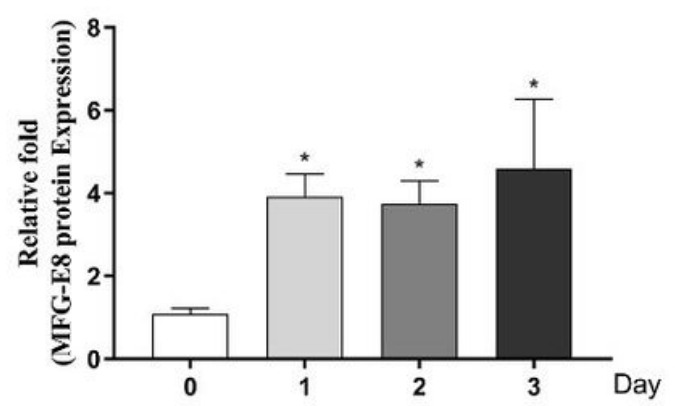

$\mathrm{C}$

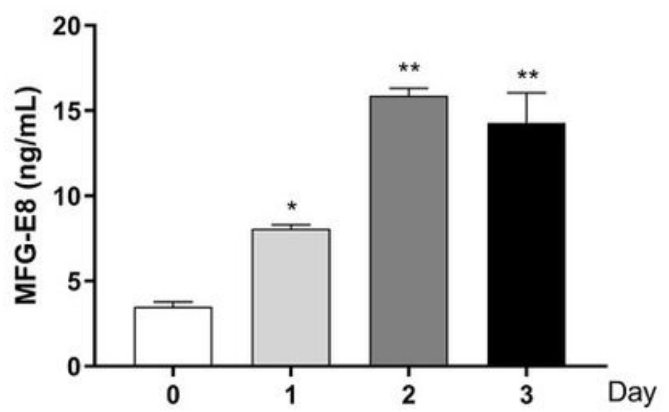

Figure 1 
Endogenous MFG-E8 expression increased significantly during osteogenic differentiation of hBMSCs. (A) Relative protein expression levels of endogenous MFG-E8 were measured by western blot after osteogenic differentiation on days 1,2, and 3 . The protein expression levels were normalized relative to that of GAPDH protein. (B) Relative mRNA expression of endogenous MFG-E8 measured by RT-PCR after 1,2 and 3 days of osteogenesis. The mRNA expression levels were normalized relative to that of GAPDH RNA. (C) The levels of secretory MFG-E8 protein were measured by ELISA during the osteogenic differentiation of hBMSCs on day 1,2 and 3. Reactions were performed in triplicate. ${ }^{*} p<0.05,{ }^{*} p<0.01$ compared to the control group. 
A

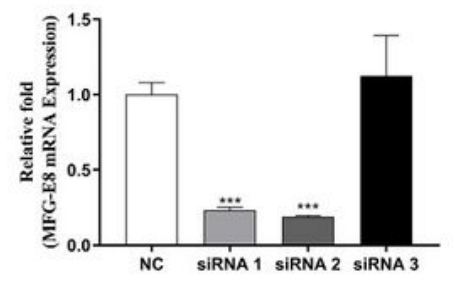

B

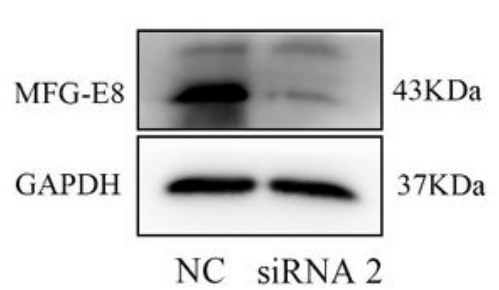

C

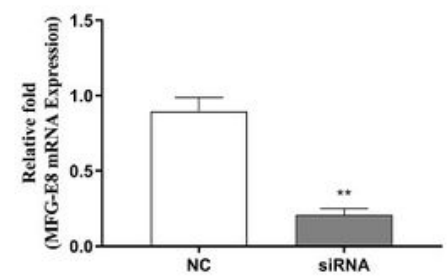

D
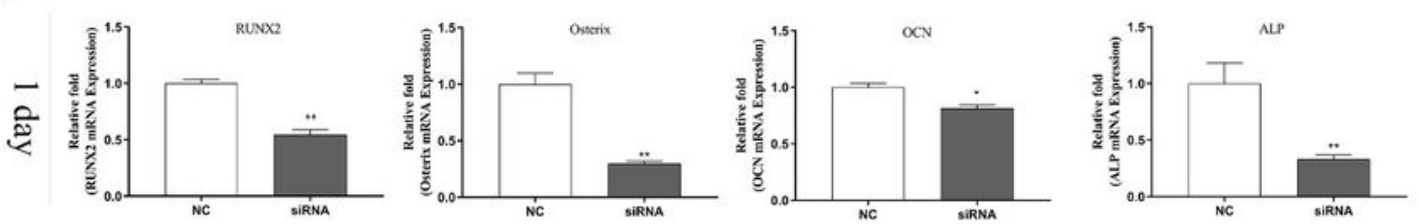

E
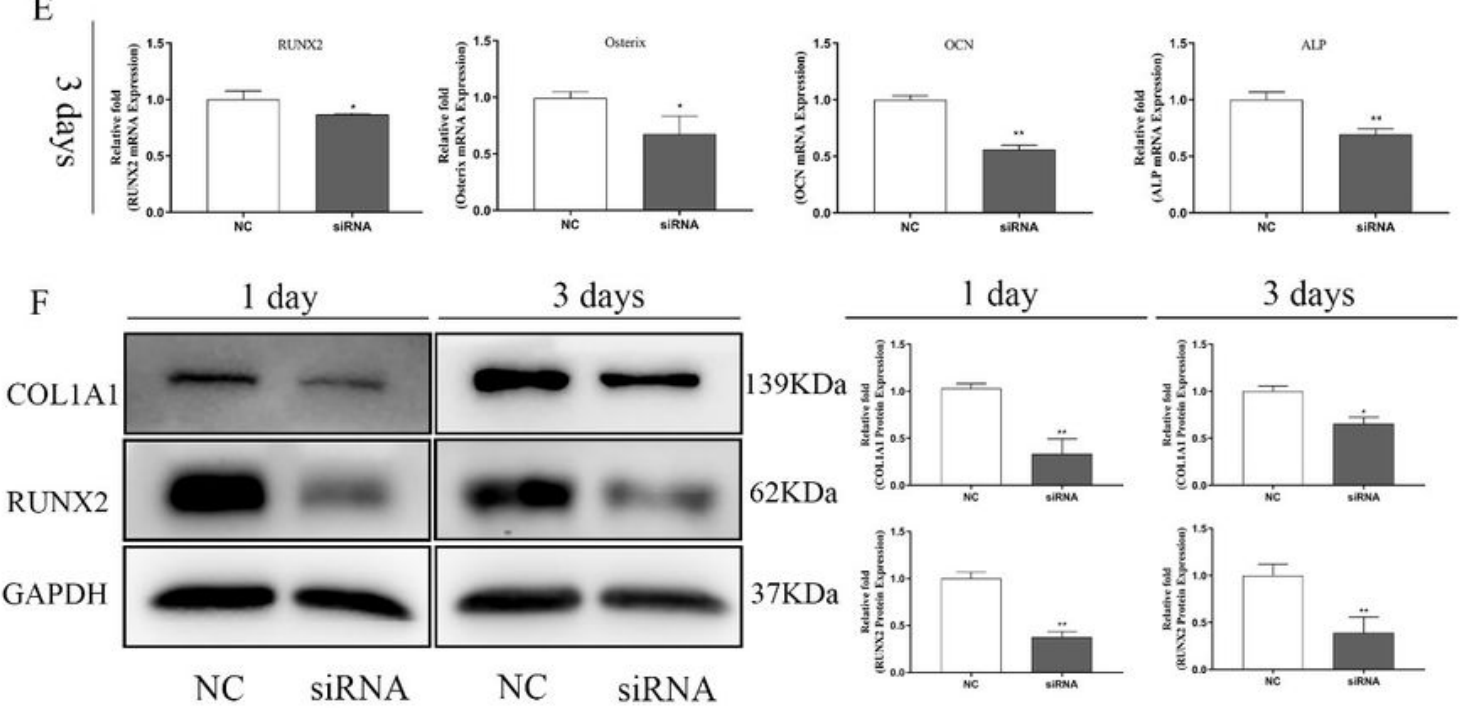

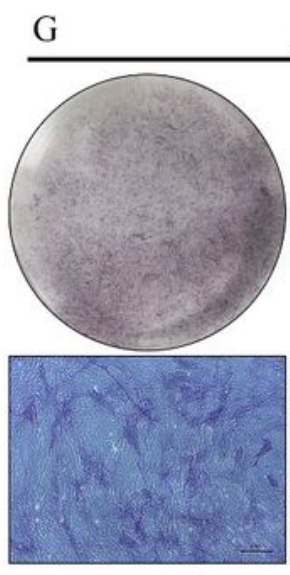

$\mathrm{NC}$
ALP
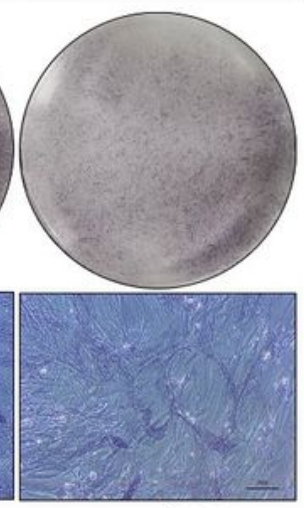

siRNA

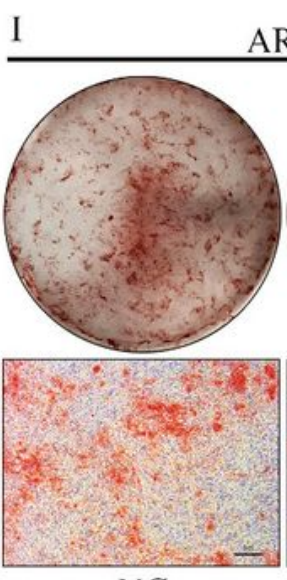

$\mathrm{NC}$ ARS
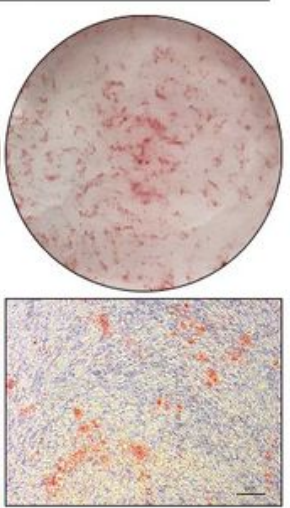

siRNA

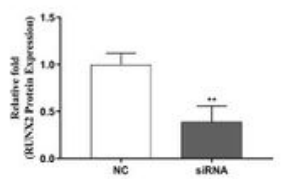

$\mathrm{H}$

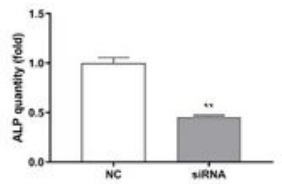

J

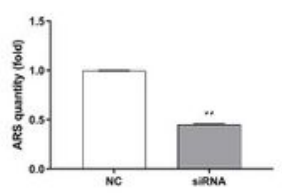

\section{Figure 2}

Knockdown of MFG-E8 inhibited the osteogenic differentiation of hBMSCs and decreased calcium deposition. (A-B) Verification of MFG-E8 knockdown in hBMSCs by siRNA 2 compared NC group. (C-D) Relative mRNA expression of osteo-related genes (RUNX2, Osterix, ALP and OCN) measured by RT-PCR after 1 and 3days of osteogenesis. (E-F) Relative protein expression of osteo-related genes (COL1A1 and RUNX2) measured by western blotting after 1 and 3days of osteogenesis. (G) ALP staining and ARS 
staining after 3 and 11 days of osteogenesis. Scar bar, $500 \mu \mathrm{m}$. All data are expressed as the means \pm SD. Reactions were performed in triplicate. ${ }^{\star} p<0.05,{ }^{\star \star} p<0.01$ compared to the control group.
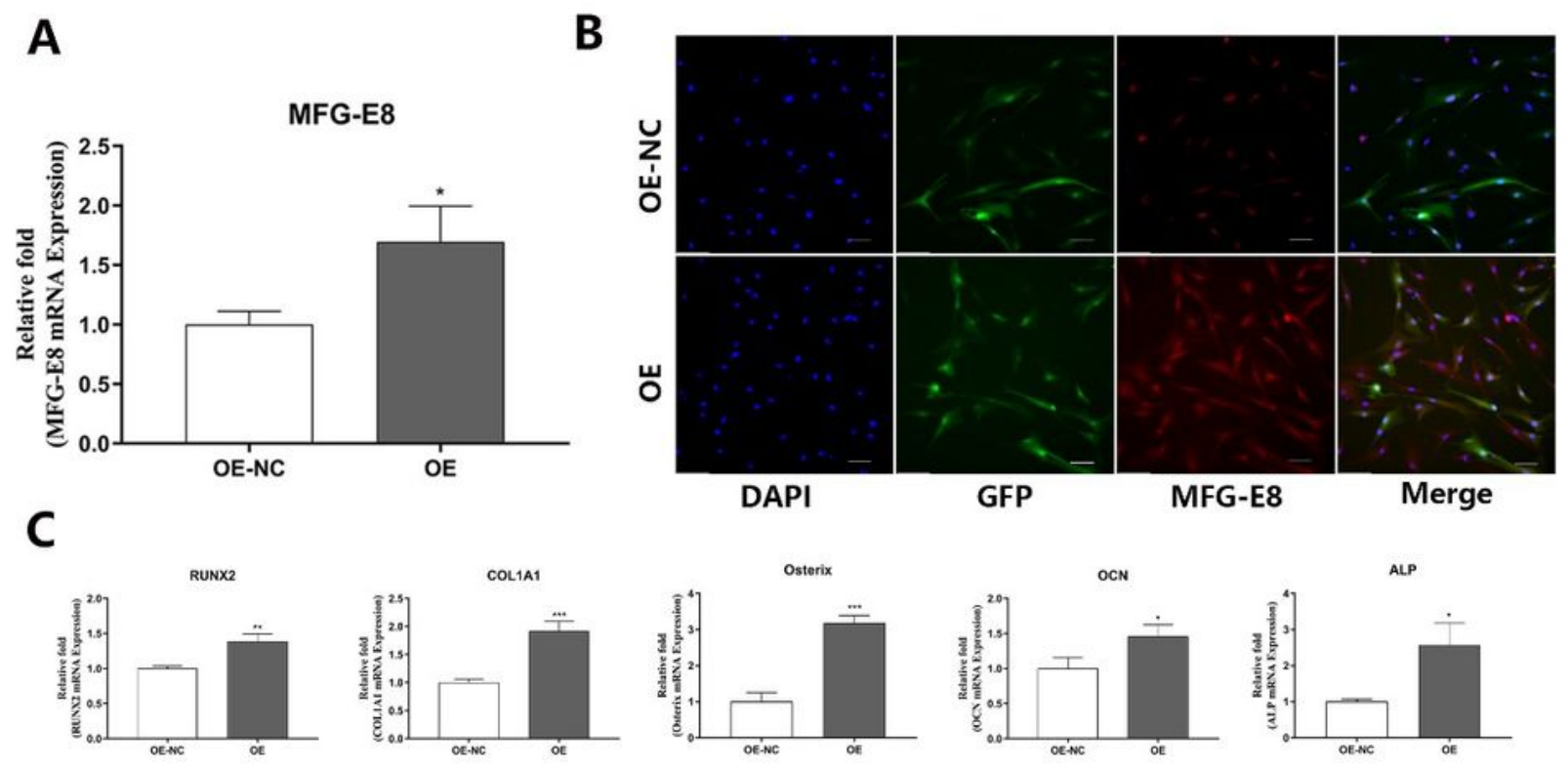

D
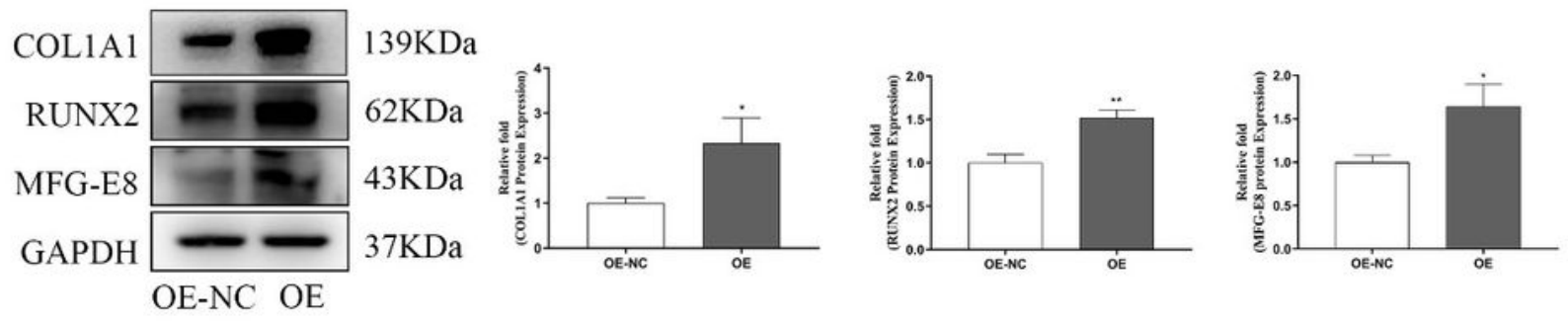

E
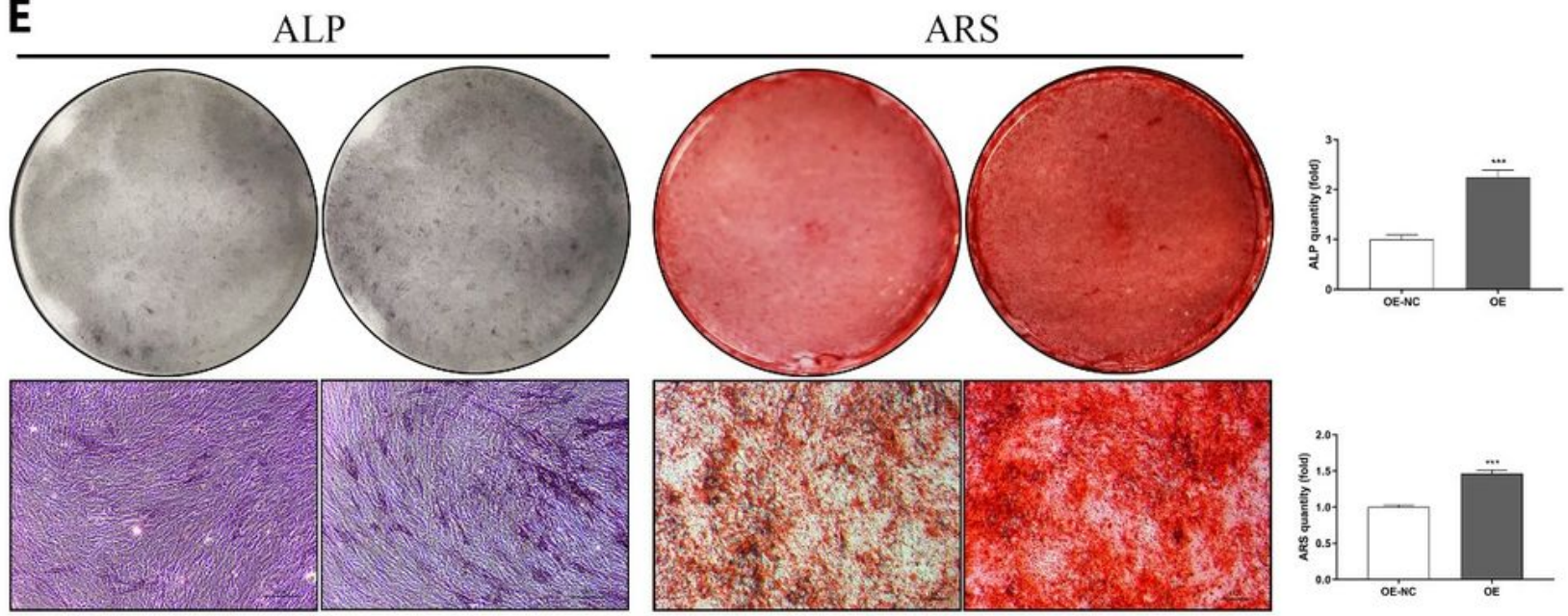

OE

\section{Figure 3}

MFG-E8 overexpression increased the levels of osteogenesis-related proteins and enhanced ALP activity and calcium deposition. (A-B) Verification of MFG-E8 overexpression in hBMSCs by lentiviral transfection compared OE-NC group. (C-D) Relative expression of osteo-related genes and proteins measured by RT- 
PCR and western blotting after 1 day of osteogenesis. (E) ALP staining and ARS staining after 3 and 14 days of osteogenesis. Scar bar, $500 \mu \mathrm{m}$. All data are expressed as the means \pm SD. Reactions were performed in triplicate. ${ }^{*} p<0.05,{ }^{* *} p<0.01$ compared to the control group.
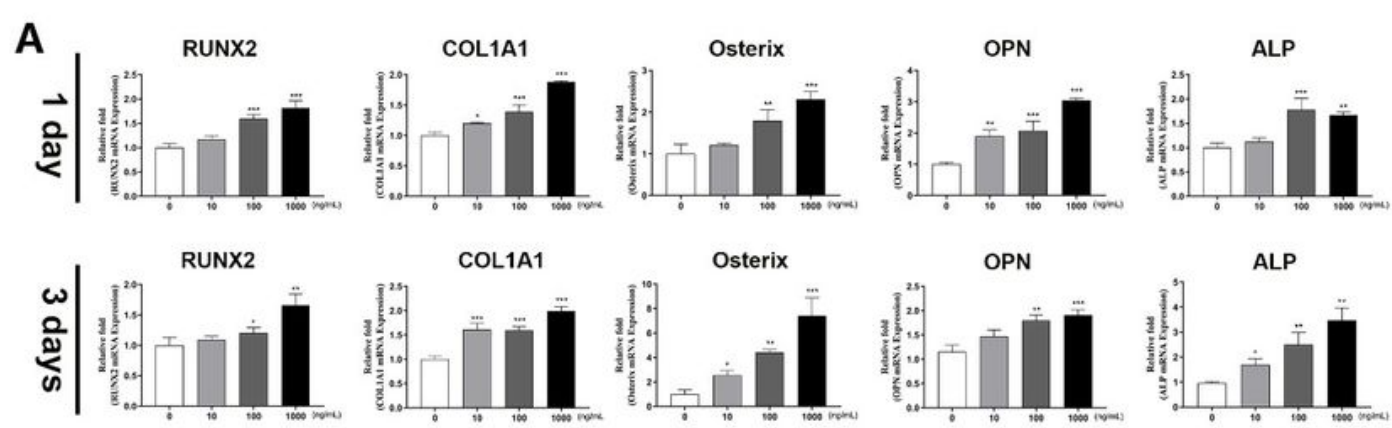

B

1 day

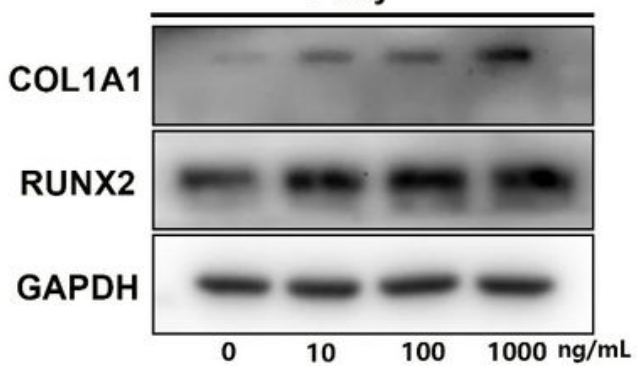

3 days
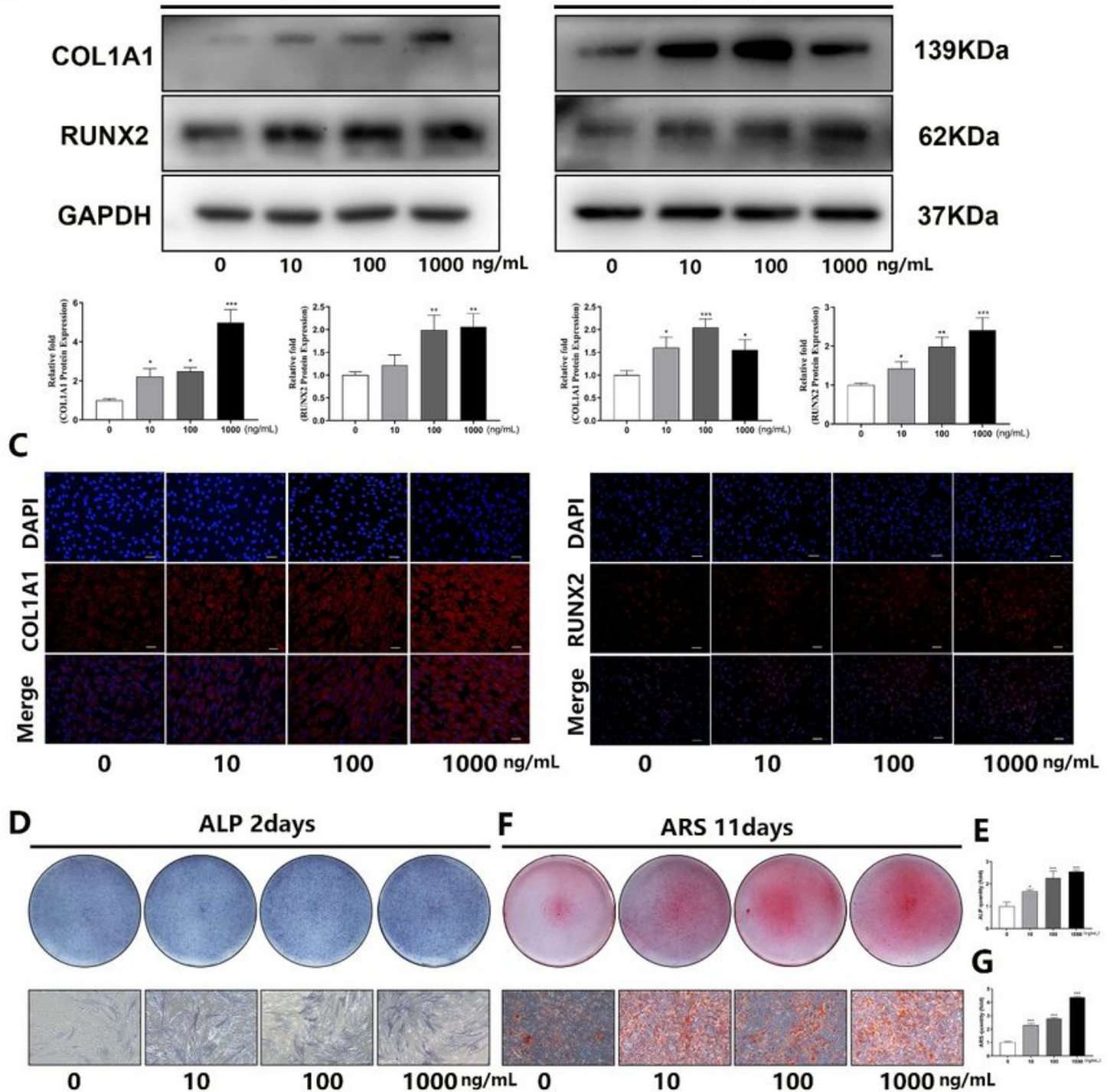

Figure 4

Exogenous MFG-E8 increased the expression levels of osteogenesis-related genes and proteins and enhanced calcium deposition. (A-B) Relative expression of osteo-related genes and proteins was 
measured by RT-PCR and western blot after treatment with various concentration of rhMFG-E8 on day 1 and 3 of osteogenesis. (C) Immunofluorescence staining for COL1A1 and RUNX2 protein after 1 day of osteogenesis. Scale bars, $100 \mu \mathrm{m}$. (D) ALP staining and quantitative assay on day 2 of osteogenic differentiation. Scale bar, $500 \mu \mathrm{m}$. (E) Mineralization was measured by ARS staining and quantitative assay after 11 days of osteogenesis. Scar bar, $500 \mu \mathrm{m}$. All data are expressed as the means \pm SD. Reactions were performed in triplicate. ${ }^{*} p<0.05,{ }^{*} p<0.01$ compared to the control group.

A

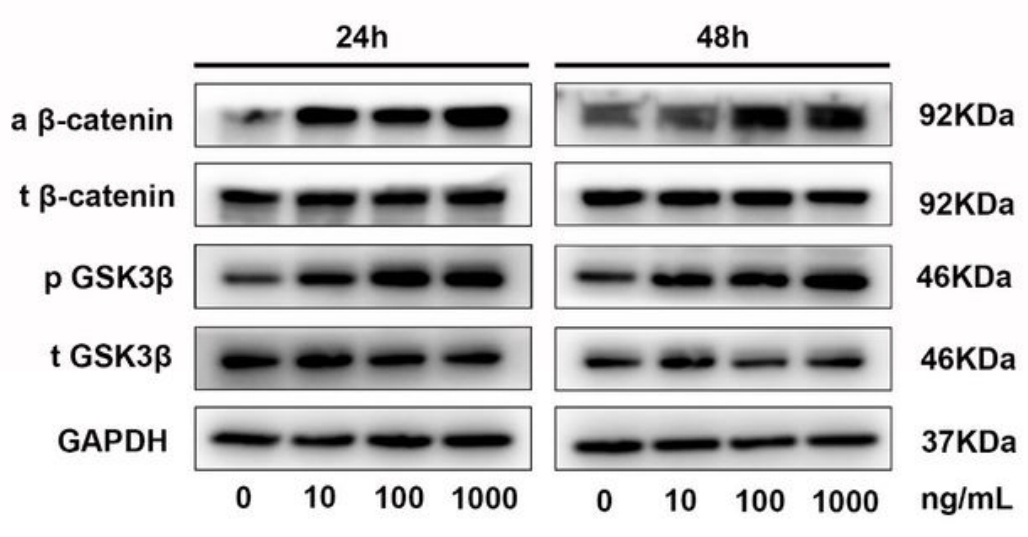

B
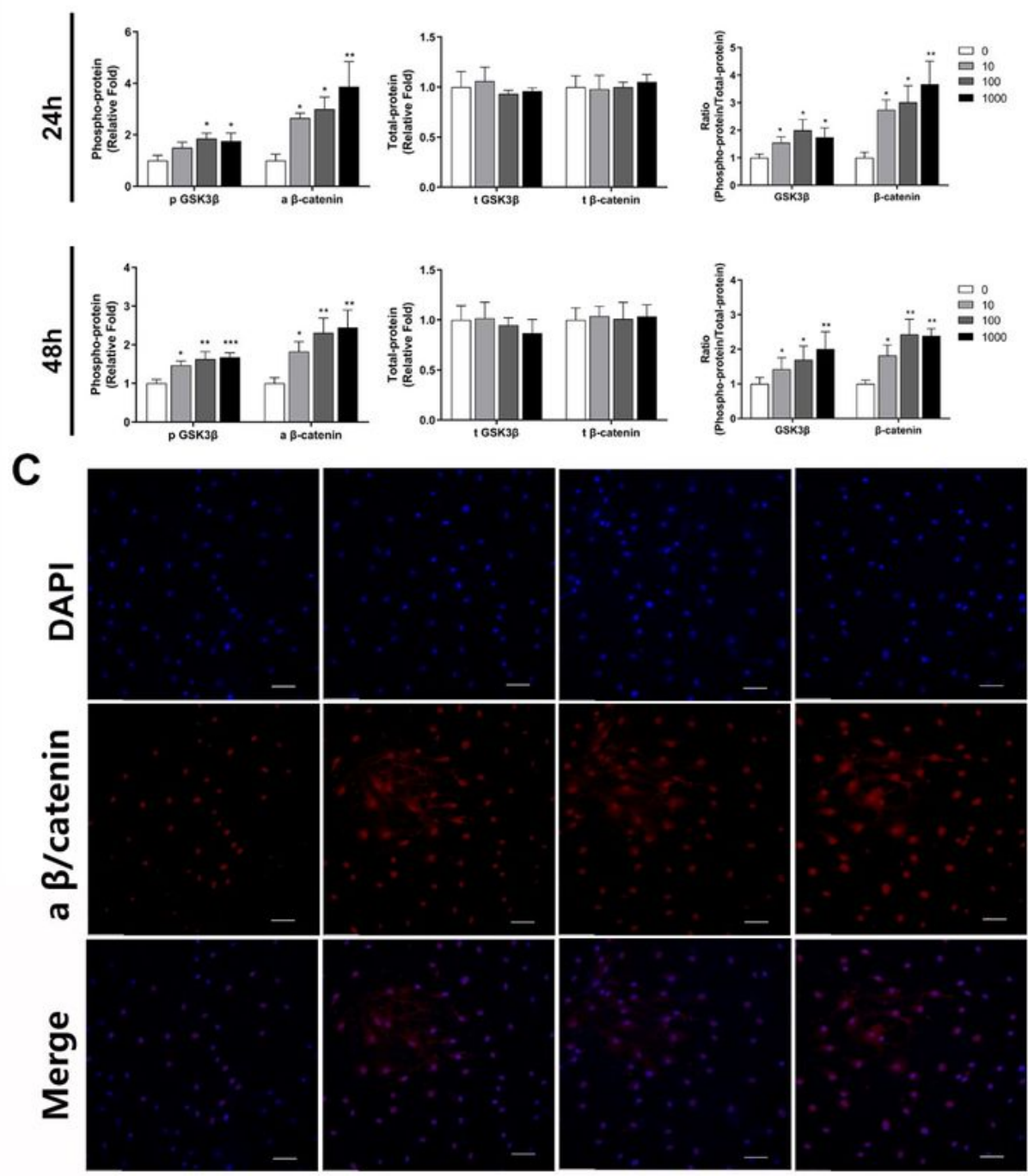

Figure 5 
MFG-E8 promotes the osteogenic differentiation of hBMSCs via the GSK3 $\beta / \beta$-catenin signaling pathway. (A-B) Relative expression of $\mathrm{Wnt} / \beta$-catenin signaling pathway related proteins was measured by western blot after treatment with various concentration of rhMFG-E8 on day 1 and 2 of osteogenesis. (C) Immunofluorescence staining for active $\beta$-catenin protein after 1 day of osteogenesis. Scale bars, 200 $\mu \mathrm{m}$. All tests were performed in triplicate. Data are expressed as means \pm SD. ${ }^{\star \star} p<0.01$ compared to the control group.
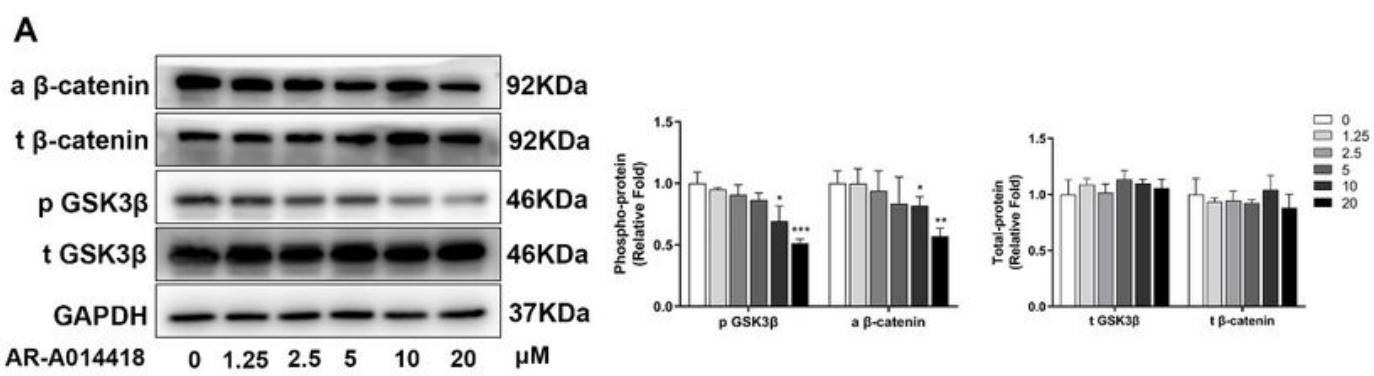

B
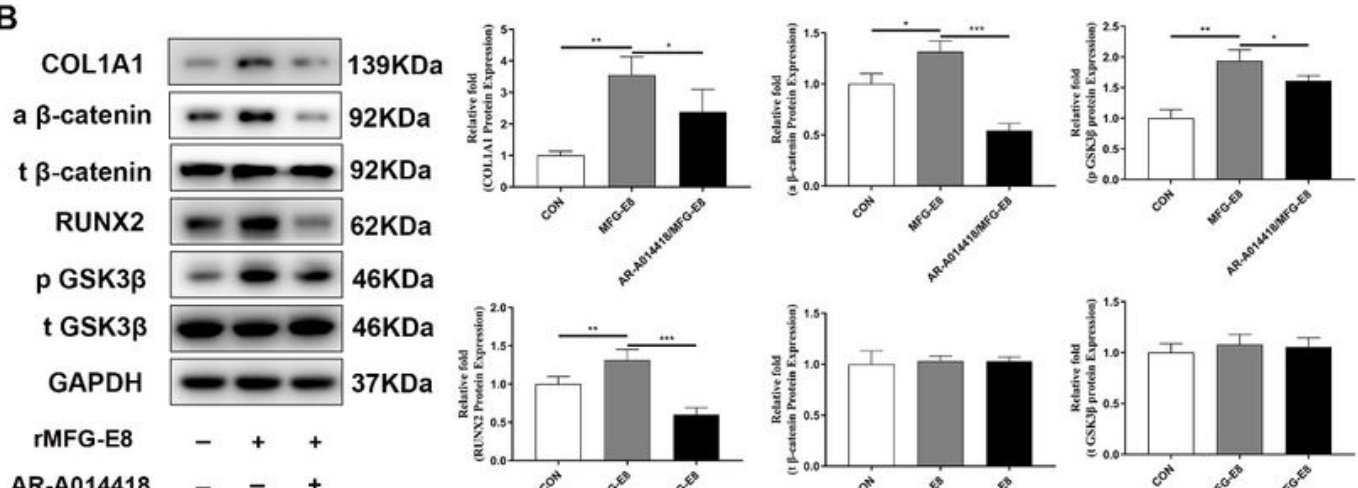

AR-A014418 - - +

C
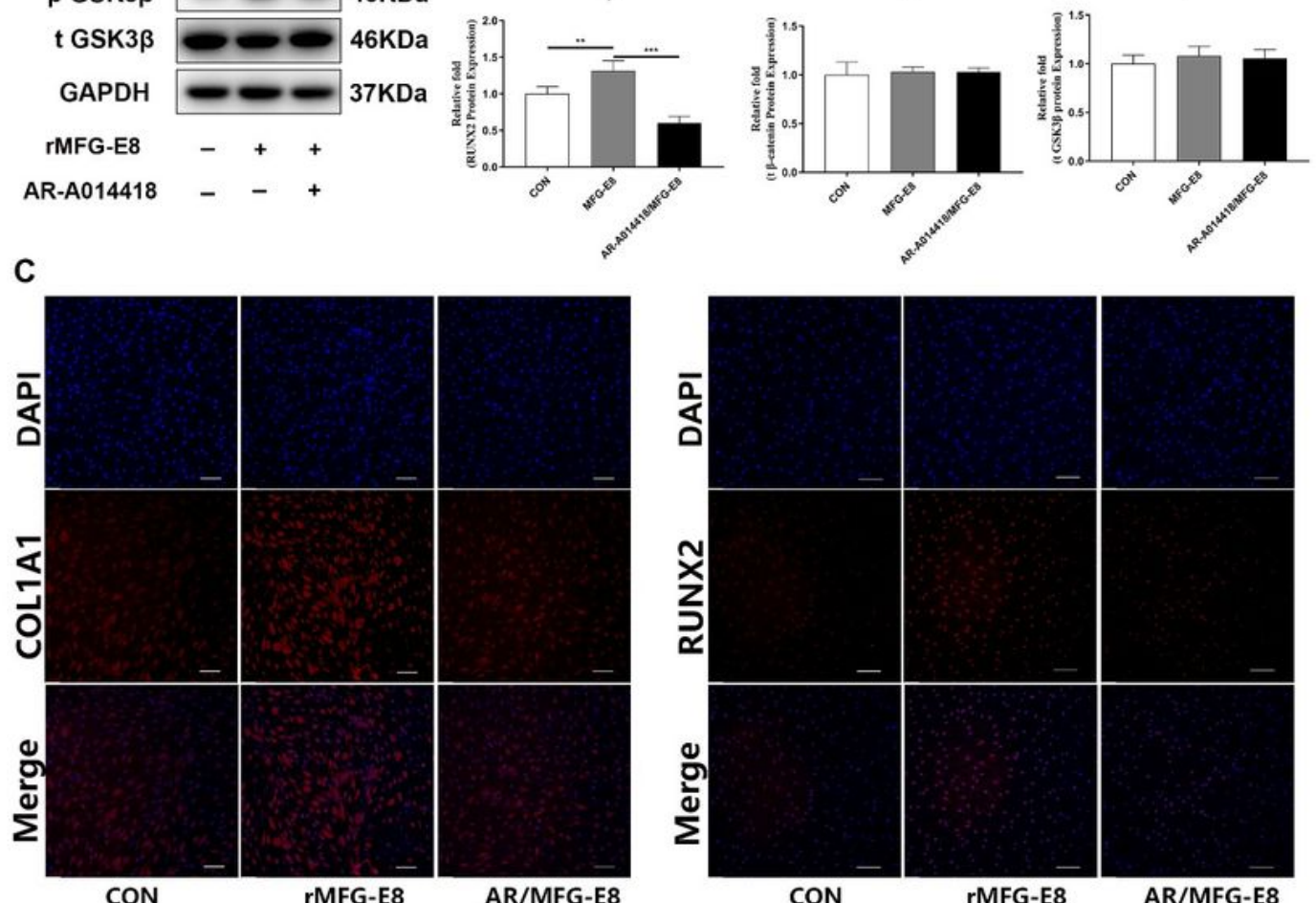

D
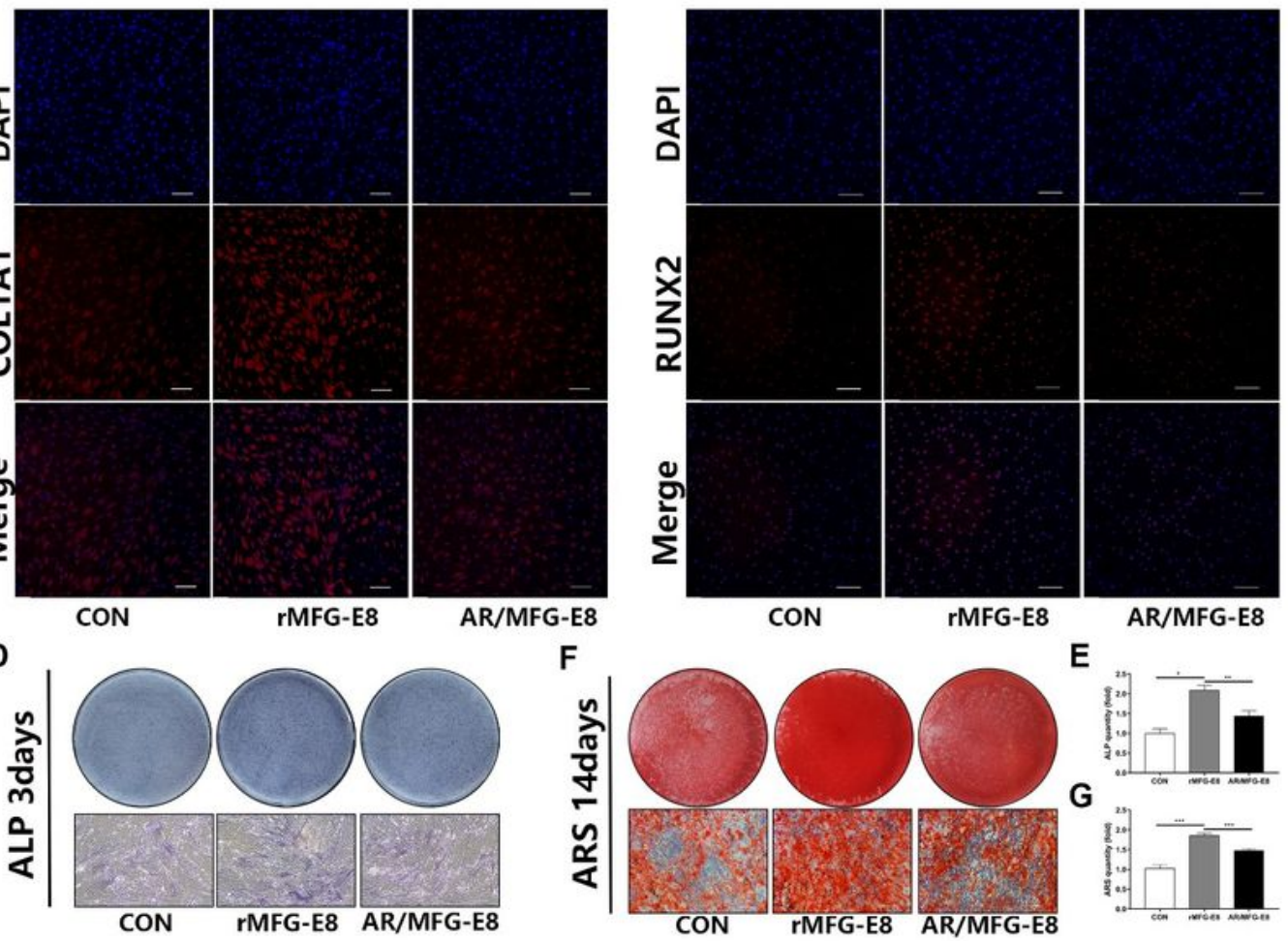

E

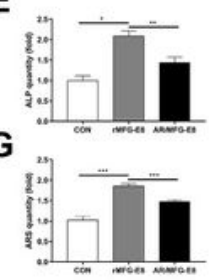

Figure 6 
Enhanced osteogenic differentiation of hBMSCs caused by MFG-E8 was partially attenuated by a GSK3 $\beta / \beta$-catenin signaling inhibitor. (A) Determination of suitable concentration of inhibitor. (B) Relative expression of proteins was measured by western blot after treatment with rhMFG-E8 $(1000 \mathrm{ng} / \mathrm{ml})$ and AR-A014418 $(20 \mu M)$ on day 1 of osteogenesis. (C) Immunofluorescence staining for RUNX2 and COL1A1 protein after 1 day of osteogenesis. Scale bars, $100 \mu \mathrm{m}$. (D) ALP staining and quantitative assay on day 3 of osteogenic differentiation. Scale bar, $500 \mu \mathrm{m}$. (E) Mineralization was measured by ARS staining and quantitative assay after 14 days of osteogenesis. Scar bar, $500 \mu \mathrm{m}$. Data are expressed as means \pm SD. ${ }^{*} p<0.05$ and ${ }^{* *} p<0.01$ between the two groups. 


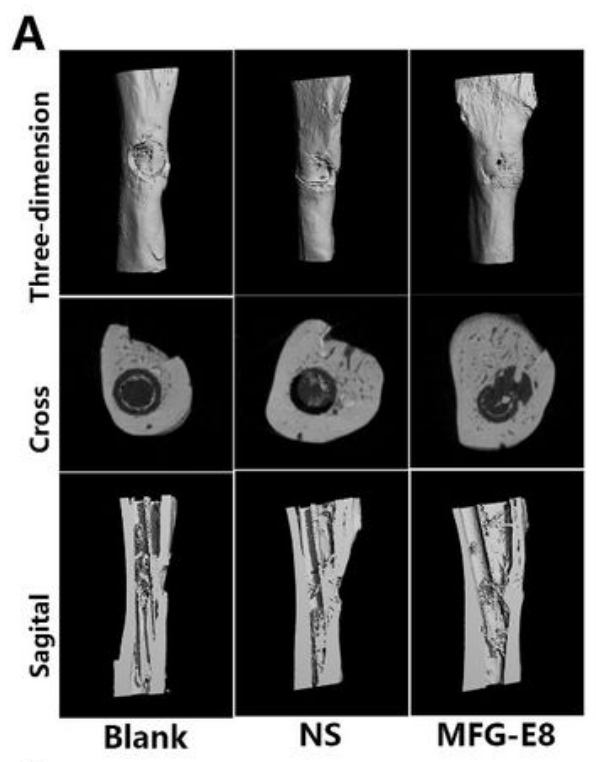

B
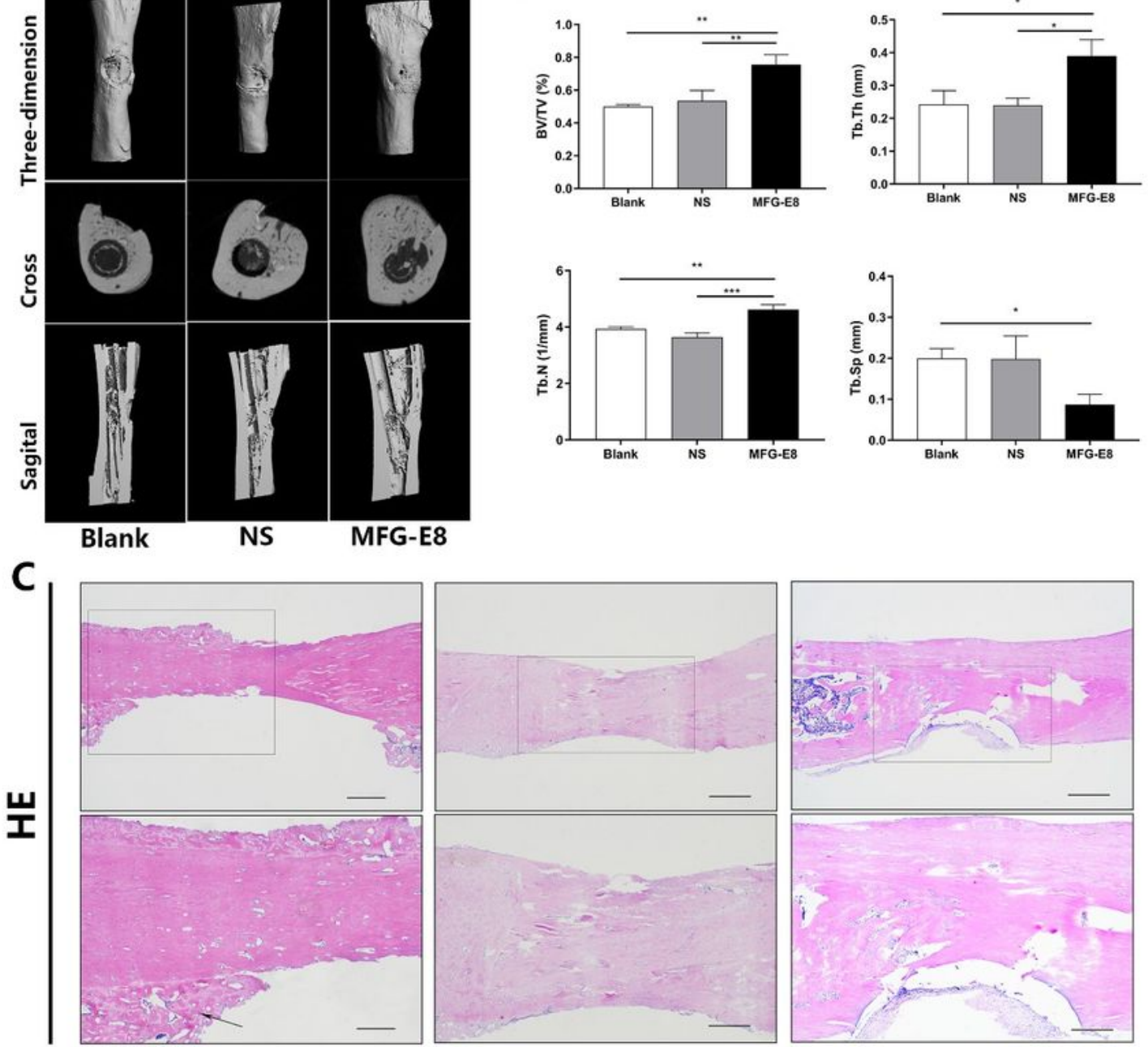

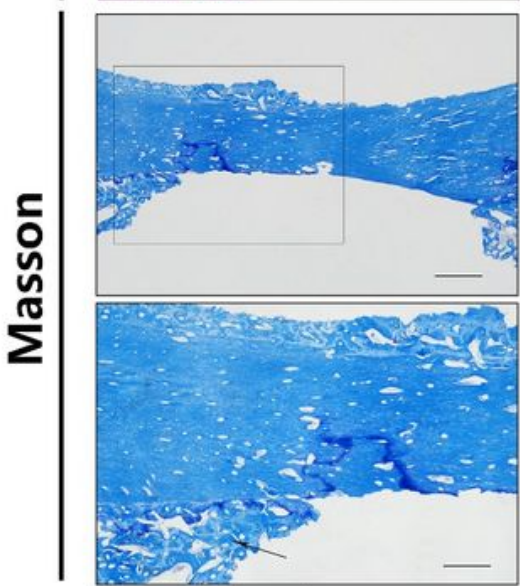

Blank

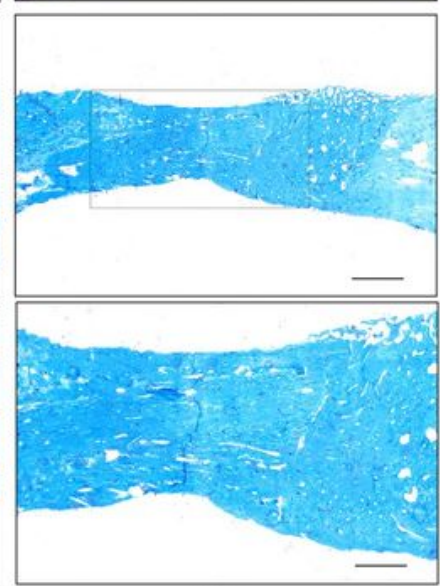

NS

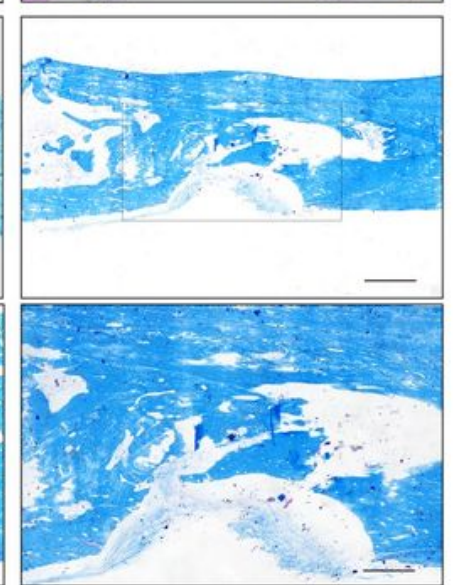

MFG-E8

Figure 7

MFG-E8 accelerated bone healing in a rat tibial-defect model. (A) Micro-CT analysis of bone fracture healing. (B) BV/TV, Tb.N, Tb,Th and Tb.Sp were analyzed by micro-CT. All experiments were performed in triplicate. (C) Histological evaluation, including HE and Masson's trichrome of mouse tibia defect area at 1 month after surgery. Scale bar, $500 \mu \mathrm{m}$. Data are expressed as means \pm SD. ${ }^{*} p<0.05$ and ${ }^{* \star} p<0.01$ between two groups. 


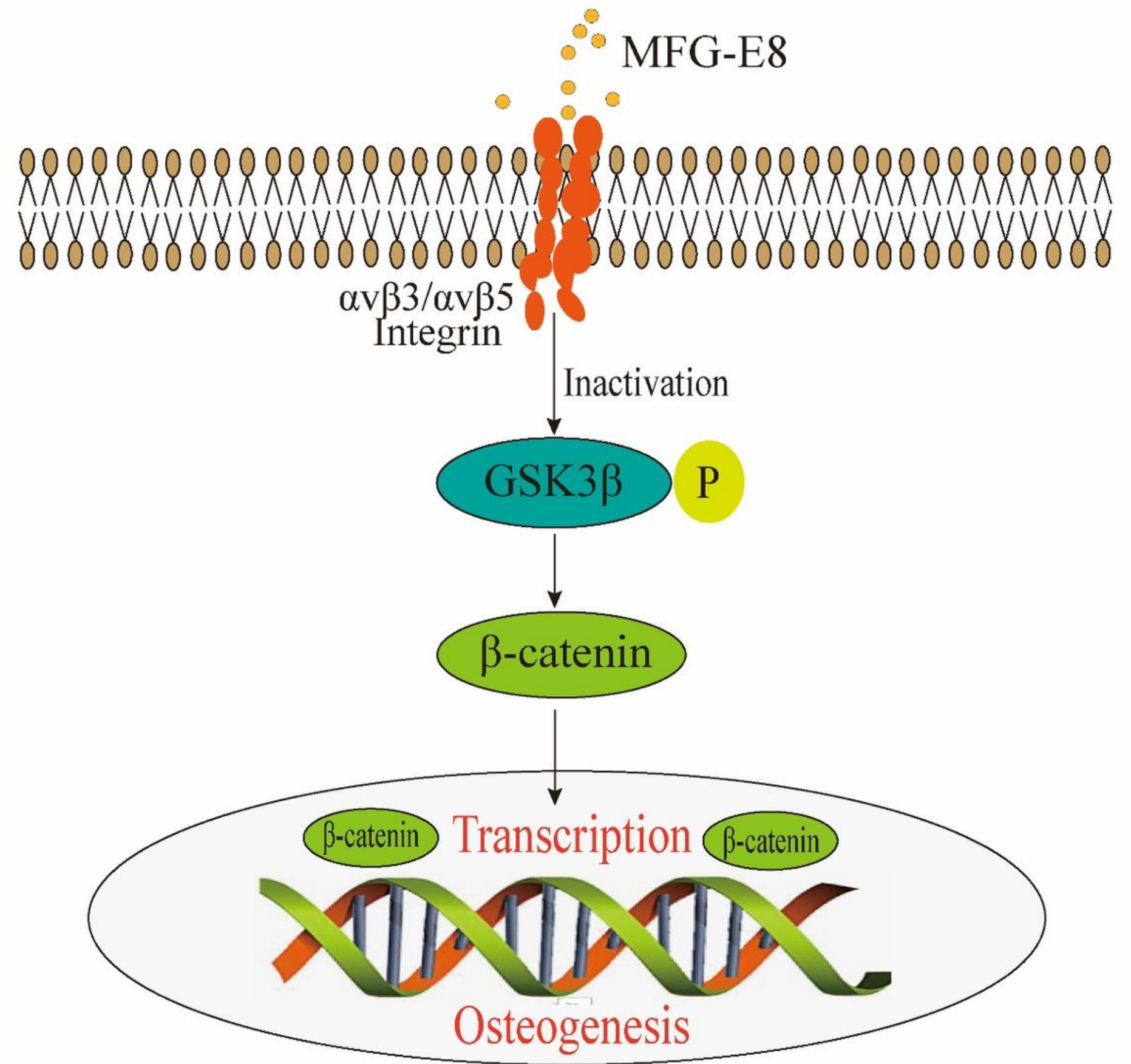

Figure 8

Schematic representation of the research presented in this figure.

\section{Supplementary Files}

This is a list of supplementary files associated with this preprint. Click to download.

- Supplementalfile.pdf 